\title{
Identification of TAZ as the essential molecular switch in orchestrating SCLC phenotypic transition and metastasis
}

Yujuan Jin ${ }^{1,2,3}$, Qiqi Zhao ${ }^{1,2,3,10,16}$, Weikang Zhu ${ }^{4,16}$, Yan Feng ${ }^{1,2,3,16}$, Tian Xiao ${ }^{5,16}$, Peng Zhang ${ }^{6}$, Liyan Jiang $^{7}$, Yingyong Hou ${ }^{8}$, Chenchen Guo ${ }^{1,2,3}$, Hsinyi Huang ${ }^{1,2,3}$, Yabin Chen ${ }^{1,2,3,10}$, Xinyuan Tong $^{1,2,3}$, Jiayu Cao ${ }^{1,2,3}$, Fei Li ${ }^{9}$, Xueliang Zhu ${ }^{1,2,3}$, Jun Qin ${ }^{11}$, Dong Gao ${ }^{1,2,3}$, Xin-Yuan Liu ${ }^{1,2,3}$, Hua Zhang $^{12}$, Luonan Chen ${ }^{1,2,3}$, Roman K Thomas ${ }^{13}$, Kwok-Kin Wong ${ }^{12}$, Yong Wang ${ }^{4,17}$, Liang Hu ${ }^{1,2,3,17}$, Hongbin $\mathrm{Ji}^{1,2,3,14,15,17}$

${ }^{1}$ State Key Laboratory of Cell Biology,

${ }^{2}$ Shanghai Institute of Biochemistry and Cell Biology,

${ }^{3}$ Center for Excellence in Molecular Cell Science, Chinese Academy of Sciences; Shanghai 200031, China.

${ }^{4}$ CEMS, NCMIS, MDIS, Academy of Mathematics and Systems Science, Chinese Academy of Sciences, Beijing 100190, China.

${ }^{5}$ Shenzhen Key Laboratory of Translational Medicine of Tumor, Department of Cell Biology and Genetics, Shenzhen University Health Sciences Center, Shenzhen, Guangdong, 518060, China.

${ }^{6}$ Shanghai pulmonary hospital, Tongji University, Shanghai, 200092, China.

${ }^{7}$ Shanghai chest hospital, Jiaotong University, Shanghai, 200030, China.

${ }^{8}$ Zhongshan hospital, Fudan University, Shanghai, 200032, China.

${ }^{9}$ Department of Pathology, School of Basic Medical Sciences, Fudan University, Shanghai, China. ${ }^{10}$ School of Life Science and Technology, Shanghai Tech University, Shanghai, 200120, China.

${ }^{11}$ CAS Key Laboratory of Tissue Microenvironment and Tumor, CAS Center for Excellence in Molecular Cell Science, Shanghai Institute of Nutrition and Health Sciences, Chinese Academy of Sciences, Shanghai, China.

${ }^{12}$ Laura and Isaac Perlmutter Cancer Center, New York University Langone Medical Center, New York, NY 10016, USA.

${ }^{13}$ Department of Translational Genomics, Center of Integrated Oncology Cologne-Bonn, Medical Faculty, University of Cologne, 50931 Cologne, Germany. Department of Pathology, University Hospital Cologne, 50937 Cologne, Germany.

${ }^{14}$ School of Life Science, Hangzhou Institute for Advanced Study, University of Chinese Academy of Sciences, Hangzhou 310024, China

${ }^{15}$ Leading author.

${ }^{16}$ These authors contributed equally to this work.

${ }^{17}$ Correspondence to Hongbin Ji, hbji@sibcb.ac.cn, Liang Hu, liang.hu@sibcb.ac.cn, or Yong Wang, ywang@amss.ac.cn 


\begin{abstract}
Small cell lung cancer (SCLC) is a recalcitrant cancer featured with high metastasis. However, the exact cell type contributing to metastasis remains elusive. Using $R b 1^{\mathrm{LL}} / \operatorname{Trp} 53^{\mathrm{LL}}$ mouse model, we identify the $\mathrm{NCAM}^{\mathrm{hi}} \mathrm{CD} 44^{\mathrm{lo} /}$ subpopulation as SCLC metastasizing cell (SMC), which is progressively transitioned from non-metastasizing $\mathrm{NCAM}^{\mathrm{lo}} \mathrm{CD} 44^{\mathrm{hi}}$ cell (Non-SMC). Integrative chromatin accessibility and gene expression profiling studies reveal an important role of SWI/SNF complex, and knockout of its central component, Brgl, significantly inhibits such phenotypic transition and metastasis. Mechanistically, TAZ is silenced by SWI/SNF complex during SCLC malignant progression, and its knockdown promotes SMC transition and metastasis. Importantly, ectopic TAZ expression reversely drives SMC-to-Non-SMC transition and alleviates metastasis. Single-cell RNA-sequencing analyses identify SMC as the dominant subpopulation in human SCLC metastasis, and immunostaining data show a positive correlation between TAZ and patient prognosis. These data uncover high SCLC plasticity and identify TAZ as key molecular switch in orchestrating SCLC phenotypic transition and metastasis.
\end{abstract}




\section{Introduction}

Small cell lung cancer (SCLC) features with very poor prognosis, with about $15 \%$ global lung cancer incidence and a five-year survival lower than $7 \%{ }^{1}$. This can be largely attributed to the highly metastatic capability of SCLC. Most SCLC patients are initially diagnosed at extensive stage, characterized with nearby lung and/or distant metastases. Therefore, it's urgently needed to explore the mechanisms involved in SCLC metastasis so as to provide helpful insights into clinical management.

Previous studies have shown that approximately $90 \%$ of human SCLC harbor concurrent inactivating mutations or deletions of $R b 1$ and $\operatorname{Trp} 53^{2}$. Homozygous deletion of these two alleles in mouse lung epithelia promotes SCLC development and dramatic metastasis, which closely recapitulates human SCLC in the clinic ${ }^{3}$. Mouse SCLC in $R b 1^{\mathrm{LL} / T r p 53^{\mathrm{LL}}}$ (RP) model typically express neuroendocrine markers including neuronal cell adhesion molecule (NCAM) and achaete-scute complex homolog 1 (ASCL1), and frequently metastasize into distant organs ${ }^{3}$. Concurrent deletion of $P 130$, an $R b$-related gene, or Pten in RP model significantly accelerates malignant progression and SCLC metastasis ${ }^{4,5}$. Moreover, up-regulated NFIB expression is found to promote SCLC metastasis through increasing the accessibility of global chromatin ${ }^{6-8}$.

SCLC are featured with high heterogeneity ${ }^{5-15}$. It is proposed that human SCLC is composed of four different subtypes based on lineage-related transcription factors including ASCL1, NEUROD1, YAP, and POU2F3 ${ }^{14}$. More recently, an inflamed SCLC subtype has been identified with a good response to immunotherapy ${ }^{16}$. Similar heterogeneity has also been found in mouse SCLC, e.g., the CD $24{ }^{\text {hi }} C D 44{ }^{\text {lo }}{ }^{2}$ PCAM ${ }^{\text {hi }}$ subpopulation from $R P$ model is identified to harbor strong capability to form tumors in allograft assay ${ }^{11}$. Moreover, mouse SCLC are found to contain the neuroendocrine (NE) and non-neuroendocrine (non-NE) subpopulations according to distinct growth patterns in culture, with the NE subtype growing as suspension and the non-NE as adhesion ${ }^{9}$. The NE cells frequently express neuroendocrine markers including NCAM, synaptophysin (SYP) and ASCL1. In contrast, the non-NE cells tend to express mesenchymal markers such as VIMENTIN and CD44 ${ }^{9}$. It has been reported that the synergetic cooperation between NE and non-NE subpopulations is necessary for SCLC metastasis whereas neither subtype could metastasize on its own ${ }^{9}$. Therefore, the exact population responsible for SCLC metastasis still remains 
unknown.

The Switch/Sucrose-Nonfermentable (mSWI/SNF) complexes, including canonical BRG1/BRM-associated factor (BAF), polybromo-associated BAF (PBAF) and non-canonical BAF (ncBAF), are essential for chromatin remodeling ${ }^{17}{ }^{18}$. All the three complexes contain a core ATPase subunit, e.g., BRG1 (Brahma/SWI2-related gene 1, also called SMARCA4), which catalyzes the hydrolysis of ATP ${ }^{18}$. Previous studies reveal that the SWI/SNF complexes tend to function as the tumor suppressor during cancer development. Consistently, a high incidence of BRG1 inactivating mutation is detected in multiple cancer types including lung cancer ${ }^{19}$. Previous studies show that BRG1 promotes cell cycle arrest and senescence through the retinoblastoma pathway in cancer cells ${ }^{20,21}$. Interestingly, recent studies have also indicated an oncogenic role of BRG1. For example, BRG1 promotes pancreatic intraepithelial neoplasia (PanIN) development and gastric cancer metastasis $22,23,24$. In SCLC, BRG1 is preferentially required for cancer progression when MAX (Myc-associated factor) is inactivated ${ }^{25}$. These findings indicate that BRG1 might function as tumor suppressor or oncogenic driver in cell type- or genetic-context dependent manner.

The Hippo pathway is initially defined as an important pathway during organ size control and function mainly through the synergetic interaction between the transcription factors TEAD1-4 and transcriptional co-activator YAP/TAZ (WWTR1) 26. The oncogenic activities of YAP/TAZ have been well documented in multiple epithelial cancers ${ }^{26-31}$. It is well-known that YAP/TAZ sustain self-renewal and tumor-initiating capability, and promote cancer malignant progression and metastasis through epithelial to mesenchymal transition (EMT) ${ }^{30}$. Latest studies also reveal that YAP/TAZ might function as a tumor suppressor ${ }^{32-34}$. For instance, YAP expression is down-regulated in breast cancer and knockdown of YAP promotes cancer cells migration and invasiveness ${ }^{35}$. Moreover, we have previously found YAP acts as the barrier for adenocarcinoma to squamous carcinoma transdifferetiation (AST) as well as lung squamous cell carcinoma progression ${ }^{33,36}$. Nonetheless, the exact role of YAP/TAZ during SCLC metastasis has not been characterized yet.

We here identify the $\mathrm{NCAM}^{\mathrm{hi}} \mathrm{CD} 44^{\mathrm{lo} /-}$ cells as the major subpopulation responsible for SCLC metastasis. Moreover, this subpopulation is progressively transitioned from the non-metastatic $\mathrm{NCAM}^{\mathrm{lo}} \mathrm{CD} 44^{\text {hi }}$ cells via the SWI/SNF complex-mediated TAZ 
bioRxiv preprint doi: https://doi.org/10.1101/2021.07.28.454244; this version posted July 29, 2021. The copyright holder for this preprint (which was not certified by peer review) is the author/funder. All rights reserved. No reuse allowed without permission.

silencing. Our data highlight the important link between epigenetically regulated TAZ and SCLC plasticity and metastasis. 


\section{Results}

\section{Identification of the $\mathrm{NCAM}^{\mathrm{hi}} \mathrm{CD} 44^{\mathrm{lo} /-}$ subpopulation as SCLC metastasizing cells}

To study SCLC heterogeneity in link with cancer malignant progression and metastasis, we first performed immunohistochemistry (IHC) staining in RP tumors using neuroendocrine marker NCAM and mesenchymal marker CD44. In primary RP tumors, we indeed observed a heterogeneous expression pattern of these two markers (Fig. 1A). We found that the percentage of $\mathrm{NCAM}^{\mathrm{hi}} \mathrm{CD} 44^{\mathrm{lo} /-}$ tumors, defined with over $50 \%$ of cancer cells highly expressing NCAM and with low or no CD44 expression ${ }^{37}$, increased with malignant progression and metastasis (Fig. 1B and Fig. S1A, Table S1). Consistently, we found that distant organ metastases such as liver and kidney metastases uniformly exhibited the $\mathrm{NCAM}^{\mathrm{hi}} \mathrm{CD} 44^{\mathrm{lo} /}$ expression pattern $($ Fig. 1A). These data indicate that the $\mathrm{NCAM}^{\mathrm{hi}} \mathrm{CD} 44^{\text {lo/- }}$ subpopulation might be responsible for SCLC metastasis.

To test this, we then used FACS sorting to isolate the $\mathrm{NCAM}^{\mathrm{hi}} \mathrm{CD} 44^{\mathrm{lo} /}$ and $\mathrm{NCAM}^{\text {lo }} \mathrm{CD} 44^{\text {hi }}$ subpopulations from primary RP tumors (Fig. 1C). Genotyping analyses confirmed the concurrent deletion of $R b 1$ and Trp53 in both subpopulations (Fig. S1B). We found that the $\mathrm{NCAM}^{\mathrm{hi}} \mathrm{CD} 44^{\text {lo/- }}$ cells grew in culture as oncosphere with suspension growth pattern (Fig. 1C), similar to classical human SCLC cell lines. In contrast, the $\mathrm{NCAM}^{\mathrm{lo}} \mathrm{CD} 44^{\text {hi }}$ cells grew as adhesion (Fig. 1C). Moreover, higher ASCL1 level was detected in the $\mathrm{NCAM}^{\mathrm{hi}} \mathrm{CD} 44^{\text {lo/- }}$ subpopulation (Fig. 1D). We then subcutaneously transplanted $5 \times 10^{6}$ cells from either $\mathrm{NCAM}^{\mathrm{hi}} \mathrm{CD} 44^{\mathrm{lo}-}$ or $\mathrm{NCAM}^{\mathrm{lo}} \mathrm{CD} 44^{\text {hi }}$ subpopulation into nude mice and waited for up to 10 weeks for distant organ metastasis analyses. Both subpopulations formed subcutaneous tumors at $100 \%$ percentage in allograft assay (Fig. S1C). In contrast, the metastasis analyses revealed a huge difference. Most mice (13 out of 15 ) from the $\mathrm{NCAM}^{\mathrm{hi}} \mathrm{CD} 44^{\mathrm{lo} /}$ group had spontaneous metastases in liver whereas only 1 out of 15 mice from the $\mathrm{NCAM}^{\mathrm{lo}} \mathrm{CD} 44^{\mathrm{hi}}$ group displayed distant metastasis (Fig. 1E-F). The liver metastases from the $\mathrm{NCAM}^{\mathrm{hi}} \mathrm{CD} 44^{\mathrm{lo} /-}$ group exhibited characteristic marker expression pattern, similar to subcutaneous tumors (Fig. 1G-H). These data demonstrate that the $\mathrm{NCAM}^{\mathrm{hi}} \mathrm{CD} 44^{\mathrm{lo} /}$ cells are mainly responsible for SCLC metastasis. We hereafter referred to the $\mathrm{NCAM}^{\mathrm{hi}} \mathrm{CD} 44^{\mathrm{lo} /}$ and $\mathrm{NCAM}^{\mathrm{lo}} \mathrm{CD} 44^{\mathrm{hi}}$ subpopulations as SCLC 
metastasizing cell (SMC) and Non-SCLC metastasizing cell (Non-SMC), respectively.

\section{Phenotypic transition from Non-SMC to SMC contributes to SCLC metastasis}

Consistent with SMC metastases tumors, the liver metastasis lesion from Non-SMC allograft assay also exhibited the $\mathrm{NCAM}^{\mathrm{hi}} \mathrm{CD} 44^{\mathrm{lo} /}$ expression pattern (Fig. 2A and Fig. S2A). We speculated that there might exist phenotypic transition from Non-SMC to SMC during SCLC malignant progression. To test this, we established Non-SMC cell line stably expressing GFP, Non-SMC-GFP, and performed subcutaneous allograft assay (Fig. 2B). Immunofluorescence (IF) staining in allograft tumors revealed that about $13 \pm 2 \%$ GFP-positive cancer cells displayed the $\mathrm{NCAM}^{\mathrm{hi}} \mathrm{CD} 44^{\mathrm{lo} /}$ pattern whereas the rest remained as Non-SMC expression pattern (Fig. 2C and Table S2). Consistently, both suspension and adhesion growth patterns were observed when these allograft tumors were cultured in vitro (Fig. 2B). To further confirm such transition, we picked single-cell clones from Non-SMC-GFP cells and performed allograft assay with the clonal Non-SMC-GFP cell lines (Fig. 2D). Similarly, we found that these subcutaneous tumors also displayed the $\mathrm{NCAM}^{\mathrm{hi}} \mathrm{CD} 44^{\mathrm{lo} /}$ pattern, ranging from $14 \pm 2 \%$ to $20 \pm 3 \%$ (Fig. 2 E and Table S2). Mixed growth pattern was also observed in culture (Fig. 2D). We further isolated the transitioned SMC with $\mathrm{NCAM}^{\mathrm{hi}} \mathrm{CD} 44^{\mathrm{lo} /}$ pattern and tested their metastasis capability using allograft assay. In contrast to no overt metastasis in the Non-SMC group, multiple distant organ metastases, e.g., lymph node, lung and liver metastases, were detectable in the transitioned SMC group (Fig. 2F-G and Fig. S2B). We found that the liver metastases also displayed the $\mathrm{NCAM}^{\mathrm{hi}} \mathrm{CD} 44^{\mathrm{lo} /-}$ pattern (Fig. 2G). These data together convincingly proved the transition from Non-SMC to SMC and highlighted the important role of such phenotypic transition in SCLC metastasis.

\section{Brg1 knockout inhibits SMC phenotypic transition and SCLC metastasis}

To further explore the molecular mechanisms underlying Non-SMC-to-SMC transition, we performed RNA Sequencing and comparatively analyzed the gene expression profiling of SMC and Non-SMC. Small cell neuroendocrine (SCN) signature is recently established as an important index for SCLC metastasis ${ }^{38}$. Interestingly, we found a significant enrichment of SCN signature-related pathways in 
SMC whereas non-SCN related pathways (immune-related pathways) were enriched in Non-SMC (Fig. 3A, Table S3-4). Real-time PCR data further confirmed the increased expression of SCN signature genes including Ascl1, Insm1, Neurod1, Chga, Soxl1 and Ttfl in SMC (Fig. 3B). These data might partially explain the high metastasis capability of SMC.

Epigenetic alterations have been implicated in cancer plasticity ${ }^{39,40}$. We then performed the assay for transposase-accessible chromatin with next-generation sequencing (ATAC-seq) to determine the global chromatin accessibility of SMC and Non-SMC. Our analyses on transcription start sites revealed an overall reduced signal in the active promoter regions of SMC (Fig. 3C). Chromatin remodelers such as SWI/SNF complex is critical for regulating chromatin architecture and accessibility ${ }^{18}$. We found that multiple members of the SWI/SNF complex, including the central catalytic ATPase Brgl, were markedly dysregulated between these two subpopulations (Fig. S3A). Real-time PCR quantification further confirmed the significant up-regulation of Brgl in SMC (Fig. 3D).

To test whether Brgl is involved in the phenotypic transition and SCLC metastasis, we generated the $R b 1^{\mathrm{L} / \mathrm{L}} / \operatorname{Trp} 53^{\mathrm{LL}} / B r g 1^{\mathrm{L} / \mathrm{L}}$ (RPB) mouse cohort and performed comparative analyses of tumorigenesis, SCN signature enrichment and metastasis in parallel with RP model (Fig. 3E). We found that Brgl knockout significantly reduced the tumor number (Fig. 3F and Fig. S3B-C). Moreover, several SCN signature-related genes including Ascl1, Ttfl and Soxll were significantly down-regulated in RPB tumors (Fig. 3G and Fig. S3B). IHC staining of NCAM and CD44 showed that the percentage of primary tumors with SMC expression pattern was also decreased in the RPB group (Fig. 3H-I, Table S5). Notably, no liver metastasis was detected in the RPB group in contrast to about $50 \%$ incidence in RP model (Fig. 3J-K). These data support that the SWI/SNF complex is important for SMC phenotypic transition and SCLC metastasis.

\section{Epigenetic silencing of TAZ by SWI/SNF complex in SMC}

To identify the downstream mediator of SWI/SNF complex in contribution to SCLC phenotypic transition and metastasis, we first constructed the dysregulated transcriptional factor (TF) network through the integrative analyses of RNA-seq and 
ATAC-seq data as previously described ${ }^{41}$. We found that Ascll and Tead2 were top-ranked TFs with highest number of dysregulated target genes in SMC and Non-SMC respectively (Fig. 4A-B, Fig. S4-5, Table S6). ASCL1 is known as the pioneering TF that initializes neuronal reprogramming and also included in the SCN biomarker genes ${ }^{42}$. TEAD family members are important TFs that function with cofactors YAP/TAZ in cancer malignant progression ${ }^{30,43,44}$. GSEA analysis revealed that the Hippo pathway was significantly enriched in Non-SMC (Fig. 4C). Moreover, Taz/Yap stood out as top hits among the dysregulated components of Hippo pathway (Fig. 4D). Using real-time PCR, we further confirmed the decreased expression of Taz/Yap in SMC vs. Non-SMC cells (Fig. 4E, Fig. S3D).

We further asked whether Taz/Yap expression was regulated by $\mathrm{Brg} 1$. We found that Brgl knockdown in SMC cells resulted in a significant up-regulation of TAZ expression whereas the expression of YAP was not notably affected (Fig. 4F-G and Fig. S3E). Such up-regulation of TAZ was also detectable in RPB tumors in comparison to RP tumors (Fig. 4H-J, Table S5). Moreover, we observed a decreased chromatin accessibility at the promoter region of Taz in SMC cells (Fig. S3F), which might explain the reduced TAZ expression (Fig. 1D). In contrast, no substantial change of the chromatin accessibility at the Yap promoter region was observed between these two subpopulations (Fig. S3F). In supporting of this, TAZ level was obviously down-regulated in primary tumors at extensive stage (Fig. S1A). Moreover, knockdown of Aridla or Arid2, another two important components of SWI/SNF complex, obviously up-regulated TAZ expression in SMC cells (Fig. S3G-H). However, YAP expression was only slightly up-regulated with Arid2 knockdown, or even down-regulated after Aridla knockdown in SMC (Fig. S3G-H). These results together demonstrate that TAZ is silenced during Non-SMC-to-SMC transition through SWI/SNF complex-mediated epigenetic reprogramming.

\section{TAZ knockdown promotes Non-SMC-to-SMC transition and accelerates SCLC metastasis}

To explore the function of TAZ in phenotype transition and SCLC metastasis, we performed Taz knockdown in Non-SMC for allograft assay (Fig. 5A). We found that Taz knockdown in Non-SMC up-regulated NCAM and down-regulated CD44 in vitro (Fig. 5B and Fig. S6A). Moreover, Taz knockdown also promoted the invasiveness in 
matrigel, the colony formation in soft agar as well as the anti-anoikis capability in Non-SMC (Fig. 5C-E). Immunofluorescence staining of allograft tumors showed that Taz knockdown promoted the appearance of $\mathrm{NCAM}^{\mathrm{hi}} \mathrm{CD} 44^{\mathrm{lo}-}$ pattern, resembling the SMC-derived tumors (Fig. 5F, Fig. S6B). Importantly, knockdown of Taz in Non-SMC promoted distant organ metastasis (Fig. 5G). IHC staining further confirmed that these metastases displayed the SMC expression pattern (Fig. 5H). These data together demonstrate that TAZ down-regulation promotes the phenotypic transition from Non-SMC to SMC and SCLC metastasis.

\section{Ectopic TAZ expression reversely promotes the transition from SMC to Non-SMC and alleviates SCLC metastasis}

To test if the phenotypic transition from Non-SMC to SMC is reversible, we ectopically expressed a constitutive activated TAZ mutant (TAZ-4SA) ${ }^{30}$ in SMC (Fig. 5I). We found that ectopic TAZ-4SA expression in SMC dramatically down-regulated NCAM and up-regulated CD44 expression in vitro, indicative of the potential reversible transition from SMC to Non-SMC (Fig. 5J, Table S7-8). Moreover, the SCN score and related genes expression also decreased after ectopic TAZ4SA expression (Fig. 5K-L, Table S7-8). Functional assays showed that TAZ-4SA expression markedly suppressed the matrigel invasiveness, colony formation in soft agar, and anti-anoikis capability of SMC (Fig. 5M-O). Immunofluorescence staining also showed that ectopic TAZ-4SA expression promoted the Non-SMC expression pattern in comparison to SMC-derived subcutaneous tumors (Fig. 5P, Fig. S6C). More importantly, ectopic TAZ-4SA expression significantly suppressed the liver metastasis of SMC (Fig. 5Q). These findings support that ectopic Taz expression promotes the reverse transition from SMC to Non-SMC and alleviates SCLC metastasis.

\section{Low TAZ level associates with SCN signature enrichment and predicts poor prognosis of SCLC patients}

To evaluate whether our findings are clinically relevant, we downloaded public RNA sequencing dataset of 112 human SCLC ${ }^{2,45}$ and analyzed the correlation between Taz and SCN signature, and single-cell sequencing data of liver metastasis ${ }^{15}$ to detect whether SMC exist in metastatic lesion, and collected 101 Chinese surgical specimens 
for prognosis analyses (Fig. 6A). Bioinformatic analyses showed that human SCLC with low TAZ expression (TAZ ${ }^{\mathrm{lo}}$ ) displayed a significantly higher SCN score (Fig. 6B, Table S9), indicative of strong metastasis capability. The SCN signature-related pathways including Positive regulation of neurotransmitter transport, Neurotransmitter secretion, and Synaptic vesicle membrane, were significantly enriched in TAZ ${ }^{\text {lo }}$ SCLC (Fig. S7A). Consistently, most SCN signature-related genes, including ASCL1, INSM1, and CHGA, were significantly increased in TAZ ${ }^{\text {lo }}$ SCLC samples (Fig. 6C). Moreover, NCAM was increased, and CD44 was decreased in TAZ ${ }^{\text {lo }}$ SCLC specimens (Fig. 6C), indicative of the SMC pattern of these samples. And TEAD2 also decreased in these TAZ ${ }^{\text {lo }}$ samples (Fig. 6C). Besides, we observed a negative correlation between the SCN signature-related genes and TAZ, and positive correlation between CD44, TEAD2 and TAZ (Fig. S7B, Table S9).

We further took advantage of the Ireland et al. single-cell RNA sequencing data derived from SCLC liver metastasis ${ }^{15}$. Interestingly, we found that most SCLC metastatic cells showed high expression of NCAM with concurrent low expression of CD44, resembling the SMC pattern (Fig. 6D). Moreover, these cells showed high expression of the SCN signature markers INSM1 and NEUROD1, similar to the SMC in RP model (Fig. 6D). Importantly, low or no TAZ expression was detected in these metastasis cells (Fig. 6D), confirming the silence of TAZ in metastasis.

Lastly, we collected a patient cohort containing 101 Chinese SCLC surgical specimens for immunostaining analyses of NCAM, CD44 and TAZ. Most of these patients were at limited stage without distant metastases. We found that high NCAM or low CD44 levels were significantly associated with worse patient overall survival (OS) (Fig. 6E, Table S10). Moreover, TAZ ${ }^{\text {lo }}$ patients also showed a worse overall survival (Fig. 6F). These data together provide strong clinical evidence in support of our findings of SMC in RP model. 


\section{Discussion}

SCLC is the most lethal form of lung cancer, characterized with highly metastatic capacity. A growing body of evidence based on mouse models has demonstrated that SCLC is highly heterogeneous with distinct subpopulations playing different roles during malignant progression and metastasis ${ }^{5-15}$. In this study, we identify the $\mathrm{NCAM}^{\mathrm{hi}} \mathrm{CD} 44^{\mathrm{lo} /}$ cells in RP model as the SCLC metastasizing cells. We further reveal that the SMCs are progressively transitioned from Non-SMCs during SCLC malignant progression and metastasis. Our data further show that the SWI/SNF complex-mediated epigenetic down-regulation of TAZ is essential for driving such phenotype transition. Moreover, TAZ activation is sufficient to drive the reverse transition from SMC to Non-SMC and thus alleviates SCLC metastasis. With the support from clinical specimen analyses, our data demonstrate that the $\mathrm{NCAM}^{\mathrm{hi}} \mathrm{CD} 44^{\mathrm{lo} /}$ cells are mainly responsible for SCLC metastasis and the SWI/SNF-TAZ axis importantly orchestrates SCLC plasticity and metastasis (Fig. 7G).

To assess the SCLC heterogeneity in RP model, we use both NE marker NCAM and mesenchymal marker CD44 to do the immunostaining and FACS analyses, and identify the $\mathrm{NCAM}^{\mathrm{hi}} \mathrm{CD} 44^{\mathrm{lo} /}$ cells as the SCLC metastasizing cells. Previous study shows that mouse SCLC cells contain both NE and non-NE subpopulations ${ }^{9}$. However, neither subpopulation alone can metastasize and a synergetic cooperation is necessary for distant organ metastasis ${ }^{9}$. In contrast, our data show that the $\mathrm{NCAM}^{\mathrm{hi}} \mathrm{CD} 44^{\mathrm{lo} /}$ cells harbor strong metastasis capability in allograft assay, and the tumors metastasize into multiple distant organs including lymph node, lung and liver. Since the SMC defined here also express the classical NE biomarkers, we reason that the $\mathrm{NCAM}^{\mathrm{hi}} \mathrm{CD} 44^{\mathrm{lo} /-}$ cells might belong to the NE subpopulation, but with higher metastasis potential. In another words, the $\mathrm{NCAM}^{\mathrm{hi}} \mathrm{CD} 44^{\mathrm{lo} /-}$ cells might represent as the highly metastatic subpopulation of the NE subtype. Future efforts looking into the heterogeneity of NE subtype will hopefully uncover more subpopulations in link to SCLC malignant progression and metastasis.

We also find that phenotypic transition from Non-SMC to SMC contributes to SCLC metastasis, which closely links cancer plasticity and malignant progression. Indeed, recent data also show, during SCLC drug resistance acquisition, that Notch signaling 
promotes the transition from NE to non-NE subtype and thus provides a niche for resisting to drug treatment ${ }^{10}$. Similar transition from the NE to non-NE subtypes have also been found in another recent study ${ }^{15}$. Metastasis and drug resistance are two major hurdles in clinical SCLC management. Understanding of molecular mechanisms involved in the phenotypic transition in these two important events will hopefully provide a solid base for the development of novel therapeutic strategy to treat SCLC in the clinic.

Through integrative analyses of gene expression profiling and chromatin accessibility, we find that the SWI/SNF complexes play an important role during Non-SMC to SMC transition. Knockout of its ATPase BRG1 inhibits such phenotypic transition and cancer metastasis, indicating an oncogenic function of SWI/SNF complex as well as BRG1 in SCLC. Previous study shows that BRG1 is important for the activation of neuroendocrine transcription ${ }^{25}$. Consistently, we observe the suppression of neuronal gene expression by BRG1 knockdown and the enrichment of SCN signature in SMC subpopulation.

We further find that TAZ is an important downstream mediator of SWI/SNF complex during SCLC phenotypic transition. Although both YAP and TAZ are significantly up-regulated in Non-SMC, only TAZ is significantly up-regulated when Brgl is knocked down in SMC. Similar findings are also observed when Aridla or Arid2 is knocked down. Consistently, Brgl knockout in RP mouse up-regulates TAZ and significantly inhibits the SMC appearance and SCLC metastasis. Moreover, we find that low TAZ expression is associated with the SCN signature enrichment. In agreement with these observations, previous studies have shown that high YAP/TAZ expression correlates with decreased NE markers ${ }^{46}$, and YAP loss defines NE differentiation ${ }^{47}$. Meanwhile, NE lineage markers are dominant in the SCN signature, which significantly associates with SCLC malignant progression and metastasis ${ }^{38,}$ ${ }^{48-50}$. Of course, considering the redundant function between YAP and TAZ, it remains possible that YAP might also regulate SCLC phenotypic transition independent of SWI/SNF complex. Future efforts will be necessary to clarify detailed regulatory mechanisms underlying YAP expression during SCLC phenotypic transition and metastasis.

Our findings from loss-of-function and gain-of-function experiments support a tumor 
suppressive role of TAZ in SCLC. YAP/TAZ are well established as oncogenic drivers. Nonetheless, accumulating evidence has recently revealed a tumor suppressive function of YAP/TAZ in multiple cancer types ${ }^{51}$. For instance, YAP resitrics Wnt signals during intestinal regeneration which results in rapid loss of intestinal crypts, and YAP loss promotes hyperplasia and microadenomas development ${ }^{52}$. In hematological cancer, low YAP level prevents nuclear ABL1-induced apoptosis and rescued YAP expression triggers cell death ${ }^{53}$. Another study shows that the growth inhibitory effect caused by LATS1/2 deletion is due to uncontrolled activation of YAP in colon cancer ${ }^{54}$. A latest report demonstrates that LATS1/2 promotes breast cancer cell growth through inhibition of YAP/TAZ ${ }^{34}$. Our findings of the tumor-suppressive function of TAZ are also supported by clinical specimen analyses. Single cell RNA sequencing data support that the cancer cells from SCLC liver metastasis mainly display the SMC expression pattern and these metastatic cells show low or no expression of TAZ. Moreover, low TAZ level is significantly associated with poor patient survival. These data together support that TAZ works as a tumor suppressor in controlling SCLC plasticity and metastasis. 


\section{Materials and Methods}

\section{RP and RPB mouse cohort generation, maintenance and analyses.}

Mice were housed in a specific pathogen-free environment at the Shanghai Institute of Biochemistry and Cell Biology, and treated in accordance with protocols conformed to the ARRIVE guidelines and approved by the Institutional Animal Care and Use Committee of the Shanghai Institutes for Biological Sciences, Chinese Academy of Sciences (approval number: IBCB0011). Conditional knockout mice including $\operatorname{Trp} 53^{\mathrm{L} / \mathrm{L}}, \mathrm{Rb}^{\mathrm{L} / \mathrm{L} 3}$ and $B r g 1^{\mathrm{LL} 55}$ alleles were generously provided by Drs. Tyler Jacks, Ronald A. DePinho and Pierre Chambon. Mice were crossed to obtain $R b 1^{\mathrm{LL}} / \operatorname{Trp} 53^{\mathrm{L} / \mathrm{L}}$ (RP) and $R b 1^{\mathrm{LL}} / \operatorname{Trp} 53^{\mathrm{LL}} / B r g 1^{\mathrm{LL}}$ (RPB) cohorts. All experimental mice were maintained on a mixed genetic background as previously described ${ }^{56}$. Mice at 6-8 weeks old were treated with Adenovirus-CMV-Cre recombinase (Ad-Cre, $2 \times 10^{6}$ p.f.u.) by intratracheal intubation ${ }^{57}$ to allow for Cre-lox mediated recombination of floxed alleles. Mouse tumors were used for immunostaining, FACS analyses, genomic DNA extraction and genotyping as previously described ${ }^{3,55}$. The primers sequences are shown in supplementary data.

\section{Statistical analysis.}

Statistical analyses were carried out using SPSS 16.0 or GraphPad Prism 5/7 software (San Diego, CA). The significance of differences was determined using two-tailed Student's $t$ test or chi-square test. Kaplan-Meier analysis with log-rank test was used to assess patients' survival between subgroups. $\mathrm{P}$ value $<0.05$ was considered to be statistically significant.

\section{Data availability.}

Sequence data have been deposited in GEO with the primary accession code GSE158091 (ATAC-seq of SMC and Non-SMC), GSE158290 (RNA-seq of SMC and Non-SMC) and GSE158293 (RNA-seq of SMC-Ctrl and SMC-TAZ-4SA).

\section{Acknowledgements.}

We thank Drs. Tyler Jacks, Ronald A. DePinho for RP mouse model and Dr. Pierre Chambon for Brgl ${ }^{L / L}$ mouse. We are grateful to Drs. Fuming Li, Xiangkun Han for 
technical assistance and Drs. Carla F Kim, Dangsheng Li, Cheng Li, Yujiang Geno Shi, Rui Fang and Nella Dost for constructive comments.

\section{Funding.}

This work was supported by the National Natural Science Foundation of China (grants 82030083 to H.J., 81871875 to L.H.); the National Basic Research Program of China (grants 2017YFA0505501 to H.J.; 2020YFA0803300 to H.J.); the Strategic Priority Research Program of the Chinese Academy of Sciences (grants XDB19020201 to H.J.); the National Natural Science Foundation of China (grants 81872312 to H.J., 82011540007 to H.J., 31621003 to H.J., 81402371 to Y.J.); the Basic Frontier Scientific Research Program of Chinese Academy of Science (ZDBS-LY-SM006 to H.J.); the International Cooperation Project of Chinese Academy of Sciences (153D31KYSB20190035 to H.J.); the Innovative research team of high-level local universities in Shanghai (SSMU-ZLCX20180500 to H.J.).

\section{Author Contributions.}

H.J. and Y.J. conceived the idea and designed the experiments. Y.J., Q.Z., Y.F., T.X., and H.H. performed all experiments and analyzed the data. W.Z., Y.W., J.C., Y.C. and L.C. performed the bioinformatics analyses. P.Z., L.J. and Y.H. provided human SCLC specimens. C.G., K.K.W., R.K.T., H.Z., X.Z., D.G., J.Q., F.L., and X.Y.L. provided technical assistance and helpful comments. H.J., Y.J. and L.H. wrote the manuscript.

\section{Competing interests.}

The authors declare that there is no conflict of interests. 


\section{Reference}

1. Gazdar, A.F., Bunn, P.A. \& Minna, J.D. Small-cell lung cancer: what we know, what we need to know and the path forward. Nat Rev Cancer 17, 725-737 (2017).

2. George, J. et al. Comprehensive genomic profiles of small cell lung cancer. Nature 524, 47-53 (2015).

3. Meuwissen, R. et al. Induction of small cell lung cancer by somatic inactivation of both Trp53 and $\mathrm{Rb} 1$ in a conditional mouse model. Cancer cell 4, 181-189 (2003).

4. Schaffer, B.E. et al. Loss of p130 accelerates tumor development in a mouse model for human small-cell lung carcinoma. Cancer research 70, 3877-3883 (2010).

5. McFadden, D.G. et al. Genetic and clonal dissection of murine small cell lung carcinoma progression by genome sequencing. Cell 156, 1298-1311 (2014).

6. Semenova, E.A. et al. Transcription Factor NFIB Is a Driver of Small Cell Lung Cancer Progression in Mice and Marks Metastatic Disease in Patients. Cell reports 16, 631-643 (2016).

7. Denny, S.K. et al. Nfib Promotes Metastasis through a Widespread Increase in Chromatin Accessibility. Cell 166, 328-342 (2016).

8. Dooley, A.L. et al. Nuclear factor I/B is an oncogene in small cell lung cancer. Genes \& development 25, 1470-1475 (2011).

9. Calbo, J. et al. A functional role for tumor cell heterogeneity in a mouse model of small cell lung cancer. Cancer cell 19, 244-256 (2011).

10. Lim, J.S. et al. Intratumoural heterogeneity generated by Notch signalling promotes small-cell lung cancer. Nature (2017).

11. Jahchan, N.S. et al. Identification and Targeting of Long-Term Tumor-Propagating Cells in Small Cell Lung Cancer. Cell reports 16, 644-656 (2016).

12. Kwon, M.C. et al. Paracrine signaling between tumor subclones of mouse SCLC: a critical role of ETS transcription factor Pea3 in facilitating metastasis. Genes \& development 29, 1587-1592 (2015).

13. $\mathrm{Wu}, \mathrm{N}$. et al. NFIB overexpression cooperates with $\mathrm{Rb} / \mathrm{p} 53$ deletion to promote small cell lung cancer. Oncotarget 7, 57514-57524 (2016).

14. Rudin, C.M. et al. Molecular subtypes of small cell lung cancer: a synthesis of human and mouse model data. Nat Rev Cancer 19, 289-297 (2019).

15. Ireland, A.S. et al. MYC Drives Temporal Evolution of Small Cell Lung Cancer Subtypes by Reprogramming Neuroendocrine Fate. Cancer cell 38, 60-78 e12 (2020).

16. Gay, C.M. et al. Patterns of transcription factor programs and immune pathway activation define four major subtypes of SCLC with distinct therapeutic vulnerabilities. Cancer cell (2021).

17. Peterson, C.L. \& Herskowitz, I. Characterization of the yeast SWI1, SWI2, and SWI3 genes, which encode a global activator of transcription. Cell 68, 573-583 (1992).

18. Mashtalir, N. et al. Modular Organization and Assembly of SWI/SNF Family Chromatin Remodeling Complexes. Cell 175, 1272-1288 e1220 (2018).

19. Mittal, P. \& Roberts, C.W.M. The SWI/SNF complex in cancer - biology, biomarkers and therapy. Nat Rev Clin Oncol 17, 435-448 (2020).

20. Wong, A.K. et al. BRG1, a component of the SWI-SNF complex, is mutated in multiple human tumor cell lines. Cancer research 60, 6171-6177 (2000).

21. Strobeck, M.W. et al. BRG-1 is required for RB-mediated cell cycle arrest. Proceedings of the National Academy of Sciences of the United States of America 97, 7748-7753 (2000).

22. Wu, Q. et al. The BRG1 ATPase of human SWI/SNF chromatin remodeling enzymes as a driver of cancer. Epigenomics 9, 919-931 (2017).

23. Tsuda, M. et al. The BRG1/SOX9 axis is critical for acinar cell-derived pancreatic tumorigenesis. J Clin Invest 128, 3475-3489 (2018).

24. Huang, L.Y. et al. SCF(FBW7)-mediated degradation of Brg1 suppresses gastric cancer metastasis. Nature communications 9, 3569 (2018).

25. Romero, O.A. et al. MAX inactivation in small cell lung cancer disrupts MYC-SWI/SNF programs and is synthetic lethal with BRG1. Cancer Discov 4, 292-303 (2014).

26. Yu, F.X., Zhao, B. \& Guan, K.L. Hippo Pathway in Organ Size Control, Tissue Homeostasis, and Cancer. Cell 163, 811-828 (2015).

27. Zanconato, F. et al. Genome-wide association between YAP/TAZ/TEAD and AP-1 at enhancers drives oncogenic growth. Nature cell biology 17, 1218-1227 (2015). 
28. Benham-Pyle, B.W., Pruitt, B.L. \& Nelson, W.J. Cell adhesion. Mechanical strain induces E-cadherin-dependent Yap1 and beta-catenin activation to drive cell cycle entry. Science 348, 1024-1027 (2015).

29. Zhao, B. et al. Cell detachment activates the Hippo pathway via cytoskeleton reorganization to induce anoikis. Genes \& development 26, 54-68 (2012).

30. Lei, Q.Y. et al. TAZ promotes cell proliferation and epithelial-mesenchymal transition and is inhibited by the hippo pathway. Molecular and cellular biology 28, 2426-2436 (2008).

31. Lau, A.N. et al. Tumor-propagating cells and Yap/Taz activity contribute to lung tumor progression and metastasis. The EMBO journal 33, 468-481 (2014).

32. Strano, S. et al. The transcriptional coactivator Yes-associated protein drives p73 gene-target specificity in response to DNA Damage. Mol Cell 18, 447-459 (2005).

33. Huang, H. et al. YAP Suppresses Lung Squamous Cell Carcinoma Progression via Deregulation of the DNp63-GPX2 Axis and ROS Accumulation. Cancer research 77, 5769-5781 (2017).

34. Ma, S. et al. Hippo signalling maintains ER expression and ER(+) breast cancer growth. Nature 591, E1-E10 (2021).

35. Yuan, M. et al. Yes-associated protein (YAP) functions as a tumor suppressor in breast. Cell Death Differ 15, 1752-1759 (2008).

36. Gao, Y. et al. YAP inhibits squamous transdifferentiation of Lkb1-deficient lung adenocarcinoma through ZEB2-dependent DNp63 repression. Nature communications 5, 4629 (2014).

37. Maues De Paula, A. et al. A diagnosis of giant cell-rich tumour of bone is supported by p63 immunohistochemistry, when more than $50 \%$ of cells is stained. Virchows Archiv 465, 487-494 (2014).

38. Balanis, N.G. et al. Pan-cancer Convergence to a Small-Cell Neuroendocrine Phenotype that Shares Susceptibilities with Hematological Malignancies. Cancer cell 36, 17-34 e17 (2019).

39. Flavahan, W.A., Gaskell, E. \& Bernstein, B.E. Epigenetic plasticity and the hallmarks of cancer. Science 357 (2017).

40. Yuan, S., Norgard, R.J. \& Stanger, B.Z. Cellular Plasticity in Cancer. Cancer Discov 9, 837-851 (2019).

41. Duren, Z., Chen, X., Jiang, R., Wang, Y. \& Wong, W.H. Modeling gene regulation from paired expression and chromatin accessibility data. Proceedings of the National Academy of Sciences of the United States of America 114, E4914-E4923 (2017).

42. Pang, Z.P. et al. Induction of human neuronal cells by defined transcription factors. Nature 476, 220-223 (2011).

43. Zhao, B., Li, L., Lei, Q. \& Guan, K.L. The Hippo-YAP pathway in organ size control and tumorigenesis: an updated version. Genes \& development 24, 862-874 (2010).

44. Zanconato, F., Cordenonsi, M. \& Piccolo, S. YAP/TAZ at the Roots of Cancer. Cancer cell 29, 783-803 (2016).

45. Rudin, C.M. et al. Comprehensive genomic analysis identifies SOX2 as a frequently amplified gene in small-cell lung cancer. Nature genetics 44, 1111-1116 (2012).

46. Horie, M., Saito, A., Ohshima, M., Suzuki, H.I. \& Nagase, T. YAP and TAZ modulate cell phenotype in a subset of small cell lung cancer. Cancer science 107, 1755-1766 (2016).

47. Ito, T. et al. Loss of YAP1 defines neuroendocrine differentiation of lung tumors. Cancer science 107, 1527-1538 (2016).

48. Borromeo, M.D. et al. ASCL1 and NEUROD1 Reveal Heterogeneity in Pulmonary Neuroendocrine Tumors and Regulate Distinct Genetic Programs. Cell reports 16, 1259-1272 (2016).

49. Osada, H., Tatematsu, Y., Yatabe, Y., Horio, Y. \& Takahashi, T. ASH1 gene is a specific therapeutic target for lung cancers with neuroendocrine features. Cancer research $\mathbf{6 5}$, 10680-10685 (2005).

50. Osborne, J.K. et al. NeuroD1 regulates survival and migration of neuroendocrine lung carcinomas via signaling molecules TrkB and NCAM. Proceedings of the National Academy of Sciences of the United States of America 110, 6524-6529 (2013).

51. Moroishi, T., Hansen, C.G. \& Guan, K.L. The emerging roles of YAP and TAZ in cancer. Nat Rev Cancer 15, 73-79 (2015).

52. Barry, E.R. et al. Restriction of intestinal stem cell expansion and the regenerative response by YAP. Nature 493, 106-110 (2013).

53. Cottini, F. et al. Rescue of Hippo coactivator YAP1 triggers DNA damage-induced apoptosis in hematological cancers. Nature medicine 20, 599-606 (2014). 
54. Pan, W.W., Moroishi, T., Koo, J.H. \& Guan, K.L. Cell type-dependent function of LATS1/2 in cancer cell growth. Oncogene 38, 2595-2610 (2019).

55. Ding, Y. et al. Chromatin remodeling ATPase BRG1 and PTEN are synthetic lethal in prostate cancer. J Clin Invest 129, 759-773 (2019).

56. Christensen, C.L. et al. Targeting transcriptional addictions in small cell lung cancer with a covalent CDK7 inhibitor. Cancer cell 26, 909-922 (2014).

57. DuPage, M., Dooley, A.L. \& Jacks, T. Conditional mouse lung cancer models using adenoviral or lentiviral delivery of Cre recombinase. Nature protocols 4, 1064-1072 (2009). 


\section{Figure legends}

\section{Figure 1. Identification of the $\mathrm{NCAM}^{\mathrm{hi}} \mathrm{CD}^{\mathrm{lo} /-}$ cells as SCLC metastaizing cells in RP mouse model.}

(A) Representative photos of HE staining, NCAM and CD44 IHC staining of primary tumors, liver and kidney metastases (met) from RP mouse model. Scale bars, $100 \mu \mathrm{m}$. (B) Statistic analyses of the $\mathrm{NCAM}^{\mathrm{hi}} \mathrm{CD} 44^{\mathrm{lo} /-}$ tumors at limited stage (no overt distant organ metastasis) and extensive stage (overt metastasis) in RP model. The $\mathrm{NCAM}^{\mathrm{hi}} \mathrm{CD} 44^{\mathrm{lo} /}$ tumors were defined when the lesions contained more than $50 \%$ cells showing $\mathrm{NCAM}^{\mathrm{hi}}$ and CD44 ${ }^{\mathrm{lo}-}$ expression. Limited stage: 87 tumors from 4 mice were analyzed; extensive stage: 98 tumors from 4 mice were analyzed. Data were shown as mean \pm S.E.M. P value was calculated by unpaired two-tailed $t$ test . (C) Flow Cytometry (FACS) analyses of primary tumors from RP mouse model using antibodies towards EPCAM, NCAM and CD44. The tumors cells without primary antibody incubation were showed as negative control (Top panels). The $\mathrm{NCAM}^{\mathrm{lo}} \mathrm{CD} 44^{\mathrm{hi}}$ and $\mathrm{NCAM}^{\mathrm{hi}} \mathrm{CD} 44^{\mathrm{lo} /}$ cells were sorted and cultured in vitro and the representative cell growth photos were shown on the right. Scale bar, $100 \mu \mathrm{m}$. (D) Western blot detection of EPCAM, NCAM, CD44, ASCL1 and TAZ expression in established $\mathrm{NCAM}^{\mathrm{lo}} \mathrm{CD} 44^{\mathrm{hi}}$ and $\mathrm{NCAM}^{\mathrm{hi}} \mathrm{CD} 44^{\mathrm{lo} /-}$ SCLC primary cell lines. (E-F) Representative photos $(\mathbf{E})$ and the incidence $(\mathbf{F})$ of liver metastasis in nude mice subcutaneously transplanted with primary $\mathrm{NCAM}^{\mathrm{lo}} \mathrm{CD} 44^{\mathrm{hi}}$ or $\mathrm{NCAM}^{\mathrm{hi}} \mathrm{CD} 44^{\mathrm{lo} /}$ cells derived from RP mouse model. Data were shown from three independent experiments ( $n=5$ mice for each experiment). The ratio of mice with liver metastasis was also indicated. P value was calculated by unpaired two-tailed $t$ test. (G-H) Representative photos of HE staining, NCAM and CD44 IHC staining of liver metastases (G), and subcutaneous tumors $(\mathbf{H})$ in nude mice transplanted with $\mathrm{NCAM}^{\mathrm{hi}} \mathrm{CD} 44^{\mathrm{lo} /}$ cell lines. Scale bars, $100 \mu \mathrm{m}$. 
Figure 2. Phenotypic transition from Non-SMC to SMC contributes to SCLC metastasis.

(A) Representative photos for HE staining, NCAM and CD44 IHC staining in the only one liver metastasis from nude mice subcutaneously transplanted with the $\mathrm{NCAM}^{\mathrm{lo}} \mathrm{CD} 44^{\mathrm{hi}}$ cell lines (Non-SMC) derived from RP mouse model. Scale bars, 100

$\mu \mathrm{m}$. (B) Experimental scheme to test phenotypic transition from Non-SMC to SMC. Primary Non-SMC was derived from RP mouse model and ectopically expressed GFP, and then used for subcutaneous transplantation in nude mice. The subcutaneous tumors were analyzed through onco-sphere formation, and NCAM and CD44 IF staining. Onco-spheres in cell culture were indicated. (C) Representative photos of NCAM and CD44 IF staining in subcutaneous tumors from nude mice transplanted with Non-SMC-GFP cells. The $\mathrm{NCAM}^{\mathrm{hi}} \mathrm{CD} 44^{\mathrm{lo} /}$ subpopulation indicated by white arrow were microscopically counted and the ratio of $\mathrm{NCAM}^{\mathrm{hi}} \mathrm{CD} 44^{\mathrm{lo} /}$ cells was indicated on the top right corner. Scale bar, $25 \mu \mathrm{m}$. Data were shown as mean \pm S.E.M. (D) Experimental scheme to test the potential phenotypic transition using single cell-derived clonal Non-SMC-GFP. The subcutaneous tumors were then analyzed through onco-sphere formation and NCAM and CD44 IF staining. Onco-spheres in cell culture were indicated. (E) Representative photos of NCAM and CD44 IF staining in clonal Non-SMC-GFP subcutaneous tumors. C1: clone \#1; C2: clone \#2. The NCAM ${ }^{\mathrm{hi}} \mathrm{CD} 44^{\mathrm{lo} /}$ subpopulation indicated by white arrow were microscopically counted and the ratio of $\mathrm{NCAM}^{\mathrm{hi}} \mathrm{CD} 44^{\text {lo/- }}$ cells was indicated on the top right corner. Scale bar, $25 \mu \mathrm{m}$. Data were shown as mean \pm S.E.M. (F) Statistical analyses of the incidence of lymph node (LN), lung and liver metastases in nude mice subcutaneously transplanted with transitioned SMC or Non-SMC, which were derived from the clonal Non-SMC-GFP subcutaneous tumors. $n=4$ mice for transitioned SMC group and $n=5$ mice for paired Non-SMC group. P values were calculated by Pearson chi-square test. (G) Representative photos of NCAM and CD44 IHC staining of mouse livers in F. The livers from paired Non-SMC showed no metastasis. Scale bar, $100 \mu \mathrm{m}$. 
Figure 3. Knockout of Brg1 in RP mouse model significantly abrogates SMC phenotypic transition and SCLC metastasis.

(A) The enrichment of small cell neuroendocrine (SCN) signature-related pathways in SMC and the enrichment of immune-related pathways in Non-SMC. NES, Normalized Enrichment Score. (B) Real-time PCR detection of SCN signature-related genes including Ascl1, Insml, Neurodl, Chga, Soxl1 and Ttfl in SMC vs. Non-SMC. Data were shown as mean \pm S.E.M. P values were calculated by unpaired two-tailed $t$ test. (C) Trend plot (top) and heat map (below) showing ATAC-seq signal over 6kb regions centered at the transcription start sites (TSS) in SMC and Non-SMC. (D) Real-time PCR detection of Brgl expression in SMC vs. Non-SMC. (E) Schematic illustration of the comparative analyses of $R b 1^{\mathrm{LL}} / \operatorname{Trp} 53^{\mathrm{LL}} \quad(\mathrm{RP})$ and $R b 1^{\mathrm{LL}} / \operatorname{Trp} 53^{\mathrm{L} L} / B r g 1^{\mathrm{LL}}$ (RPB) mice. (F) Statistical analyses of primary tumor numbers in RP and RPB mice at 32 weeks after Ad-Cre treatment. $\mathrm{n}=18$ mice for RP group, $\mathrm{n}=10$ mice for RPB group. Data were shown as mean \pm S.E.M. P value was calculated by unpaired two-tailed $t$ test. (G) Real-time PCR detection of $\operatorname{Brg} 1$ and the SCN signature-related genes in primary tumors from RP and RPB mice. $n=2$ mice for each group. Data shown as mean \pm S.E.M. P values were calculated by unpaired two-tailed $t$ test. (H) Representative photos of NCAM and CD44 IHC staining in primary tumors from RP and RPB mice at 32 weeks after Ad-Cre treatment. Scale bar, $100 \mu \mathrm{m}$. (I) Statistical analyses of the percentage of primary tumors with $\mathrm{NCAM}^{\mathrm{hi}} \mathrm{CD} 44^{\mathrm{lo} /}$ expression pattern in RP and RPB mice. The $\mathrm{NCAM}^{\mathrm{hi}} \mathrm{CD} 44^{\mathrm{lo} /}$ tumors were defined when the lesions contained more than $50 \%$ cells showing $\mathrm{NCAM}^{\mathrm{hi}}$ and CD44 ${ }^{\mathrm{lo} /-}$ expression. A total of 56 tumors from 3 RP mice and 28 tumors from 4 RPB mice were analyzed. Data were shown as mean \pm S.E.M. P value was calculated by unpaired two-tailed $t$ test. ( $\mathbf{J})$ Representative photos of NCAM and CD44 IHC staining in livers of RP and RPB mice. The livers from RPB mice contained no metastasis. Scale bar, $100 \mu \mathrm{m}$. (K) Liver metastasis incidence in RP and RPB mice at 32 weeks after Ad-Cre treatment. $n=18$ mice for RP group, $n=10$ mice for RPB group. P value was calculated by Pearson chi-square test. 


\section{Figure 4. TAZ is epigenetically silenced by the SWI/SNF complex in SMC.}

(A) Schematic illustration of the integrative analyses of RNA-seq and ATAC-seq in SMC and Non-SMC. Specific TF networks in SMC and Non-SMC were constructed according to the PECA2 model (see details in method). (B) Enriched transcription factors (TFs) in SMC and Non-SMC through integrative analyses of ATAC-seq and RNA-seq data ranked based on the numbers of dysregulated target genes. (C) Gene set enrichment analysis (GSEA) plot of Hippo signaling pathway in SMC vs. Non-SMC. (D) Heat map of RNA-seq data showing the relative expression of TFs and cofactors in Hippo pathway in SMC vs. Non-SMC. (E) Real-time PCR detection of Taz in SMC and Non-SMC. Data were shown as mean \pm S.E.M. P value was calculated by unpaired two-tailed t test. (F) Real-time PCR detection of Brgl and Taz in SMC with or without Brgl knockdown. Gapdh served as the internal control. Data were shown as mean \pm S.E.M. P values were calculated by unpaired two-tailed $t$ test. (G) Western blot detection of BRG1 and TAZ levels in SMC with or without Brgl knockdown. GAPDH served as the internal control. (H) Real-time PCR detection of Taz in primary tumors from RP and RPB mice at 32 weeks after Ad-Cre treatment. Gapdh served as the internal control. $\mathrm{n}=2$ for each group. Data were shown as mean \pm S.E.M. P value was calculated by unpaired two-tailed $t$ test. (I) Representative photos of TAZ IHC staining in primary tumors from RP and RPB mice at 32 weeks after Ad-Cre treatment. Scale bar, $100 \mu \mathrm{m}$. (J) Percentage of TAZ positive tumors in RP vs. RPB mice at 32 weeks after Ad-Cre treatment. 56 tumors from 3 RP mice and 28 tumors from 4 RPB mice were analyzed. Data were shown as mean \pm S.E.M. P value was calculated by unpaired two-tailed $t$ test. 
Figure 5. TAZ functions as a critical molecular switch in regulating the phenotypic transition and SCLC metastasis.

(A) Schematic illustration of the comparative analyses of Non-SMC with or without Taz knockdown. (B) Western blot detection of TAZ, NCAM and CD44 levels in Non-SMC with or without Taz knockdown. GAPDH served as the internal control. (C) Matrigel invasiveness of Non-SMC with or without Taz knockdown. Scale bars, 100 $\mu \mathrm{m}$. (D) Representative photos (left) and number (right) of the soft-agar colonies of Non-SMC with or without Taz knockdown. Scale bars, $100 \mu \mathrm{m}$. Data were shown as mean \pm S.E.M. P value was calculated by unpaired two-tailed $t$ test. (E) Western blot detection of cleaved caspase3 (CC3) in anti-anoikis assay of Non-SMC with or without Taz knockdown. TUBULIN served as the internal control. (F) Representative photos of NCAM and CD44 IF staining in subcutaneous tumors from nude mice transplanted with Non-SMC with or without Taz knockdown. Scale bar, $25 \mu \mathrm{m}$. (G-H) Metastasis incidence $(\mathbf{G})$ and representative photos of NCAM and CD44 IHC staining (H) in livers from nude mice transplanted by Non-SMC with or without Taz knockdown. $\mathrm{n}=6$ for each group. $\mathrm{P}$ value was calculated by Pearson chi-square test. Scale bar, $100 \mu \mathrm{m}$. (I) Schematic illustration of the comparative analyses of SMC with or without ectopic TAZ-4SA expression. (J) Western blot detection of TAZ, NCAM and CD44 levels in SMC with or without ectopic TAZ-4SA expression. GAPDH served as the internal control. (K) SCN score of SMC with or without ectopic TAZ-4SA expression. Data were shown as mean \pm S.E.M. P value was calculated by unpaired two-tailed $t$ test. (L) Real-time PCR detection of the SCN signature-related genes in SMC with or without ectopic TAZ-4SA expression. Gapdh served as the internal control. Data were shown as mean \pm S.E.M. P values were calculated by unpaired two-tailed $t$ test. (M) Matrigel invasiveness of SMC with or without ectopic TAZ-4SA expression. Scale bar, $100 \mu \mathrm{m}$. (N) Representative photos (left) and statistical analyses (right) of soft-agar colonies of SMC with or without ectopic TAZ-4SA expression. Scale bar, $100 \mu \mathrm{m}$. Data were shown as mean \pm S.E.M. $\mathrm{P}$ value was calculated by unpaired two-tailed $t$ test. (O) Western blot detection of CC3 in anti-anoikis assay of SMC with or without ectopic TAZ-4SA expression. TUBULIN served as the internal control. (P) Representative photos of NCAM and CD44 IF staining in subcutaneous tumors from nude mice transplanted with SMC with or without ectopic TAZ-4SA expression. Scale bar, $25 \mu \mathrm{m}$. (Q) Metastasis 
incidence (left) and representative photos of NCAM and CD44 IHC staining of liver metastasis (right) in nude mice transplanted with SMC with or without ectopic TAZ-4SA expression. $\mathrm{n}=6$ mice for Ctrl group, $\mathrm{n}=7$ mice for TAZ-4SA group. Scale bar, $100 \mu \mathrm{m}$. P value was calculated by Pearson chi-square test. 


\section{Figure 6. Low TAZ level is correlated with SCN signature enrichment and predicts poor prognosis of SCLC patients.}

(A) Schematic illustration of the analyses of human SCLC specimens. (B) SCN score of human SCLC specimens with high or low TAZ mRNA level. The RNA-seq data were down-loaded from public database (GSE69091 and EGAS00001000334). Data were shown as mean \pm S.E.M. P value was calculated by unpaired two-tailed $t$ test. (C) Correlation between individual SCN signature-related genes, CD44 or TEAD2 expression with high or low TAZ level in human SCLC (GSE69091 and EGAS00001000334). Data were shown as mean \pm S.E.M. P values were calculated by unpaired two-tailed $t$ test. (D) Clustering and the NCAM, CD44, TAZ, NEUROD1 and INSM1 expression of the single cell sequencing data (GSM4558305) of liver biopsy from SCLC patient. (E-F) Representative photos of NCAM, CD44 (E) or TAZ (F) IHC staining in Chinese SCLC specimens (up) and survival curves of high or low expression of NCAM, CD44 or TAZ with overall survival (OS) (below). Scale bar, $100 \mu \mathrm{m}$. P values were calculated by Kaplan-Meier analysis with log-rank test. (G) Working model illustrating the essential role of SWI/SNF complex-mediated TAZ expression in controlling the phenotypic transition from Non-SMC to SMC and SCLC metastasis. TAZ, which is epigenetically silenced by the SWI/SNF complex, functions as a critical molecular switch during the phenotypic transition from Non-SMC to SMC and SCLC metastasis. Disruption of SWI/SNF complex through BRG1 knockout promotes TAZ up-regulation and thus inhibits the phenotypic transition and cancer metastasis. 
Figure 1

A

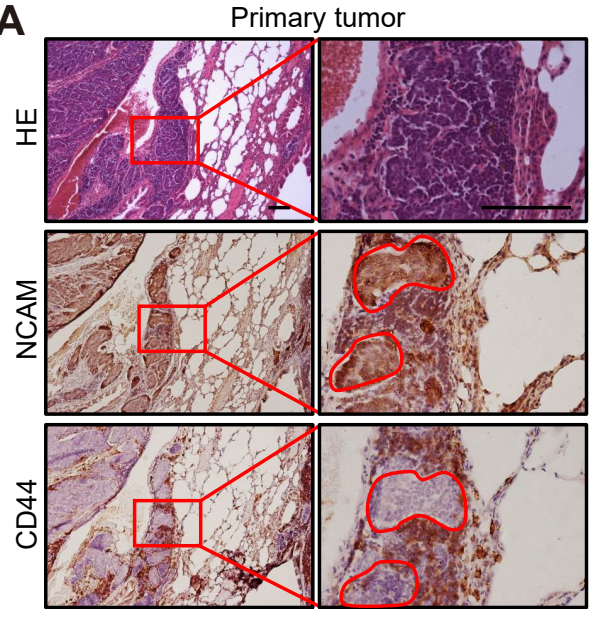

Liver met

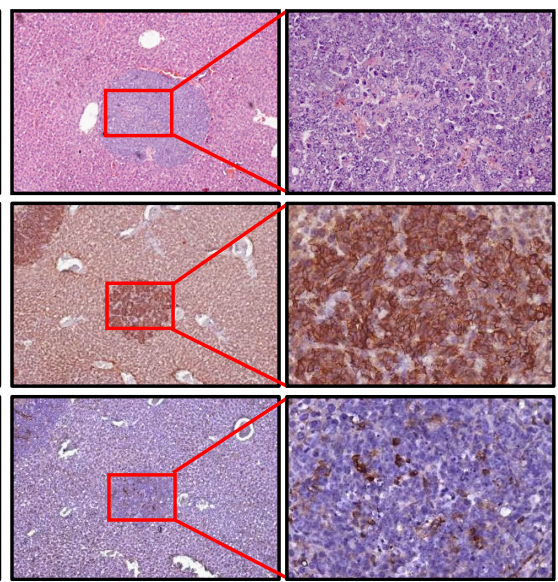

Kidney met

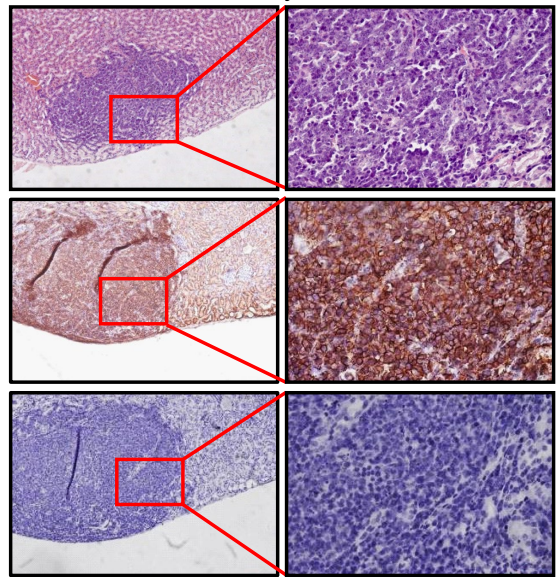

B

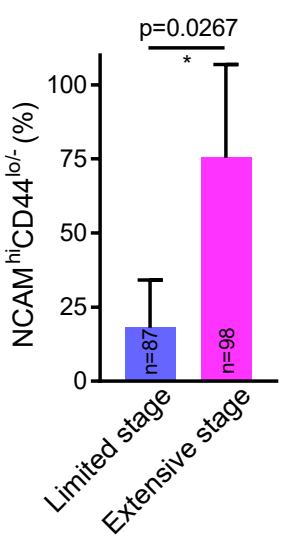

D

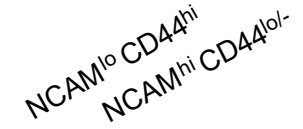

C
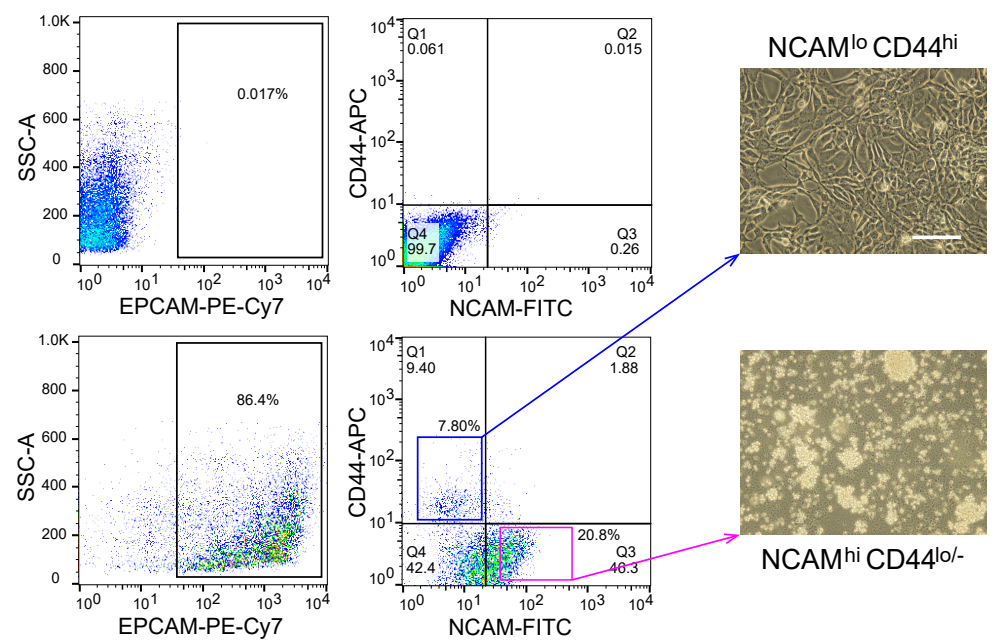

F

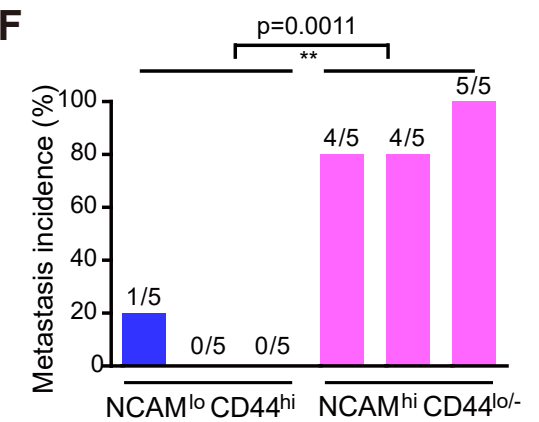

G

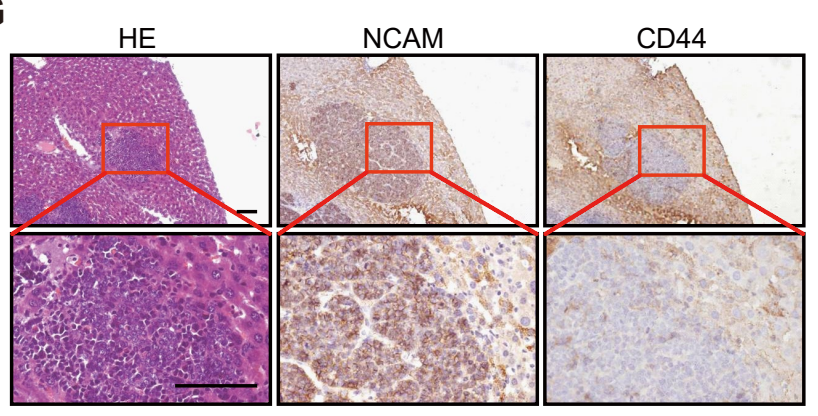

Liver met (NCAM ${ }^{\text {hi }}$ CD44/o/-)
H

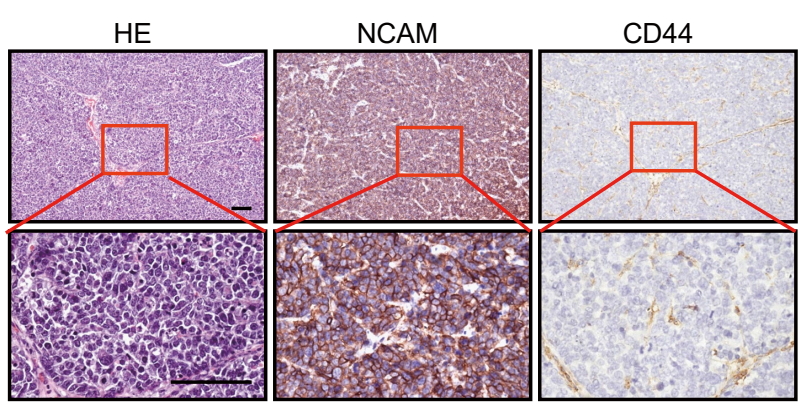

Subcutaneous tumors (NCAMhi CD44/o/-) 
Figure 2

bioRxiv preprint doi: https://doi.org/10.1101/2021.07.28.454244; this version posted July 29, 2021. The copyright holder for this preprint (which was not certified by peer review) is the author/funder. All rights reserved. No reuse allowed without permission.

A

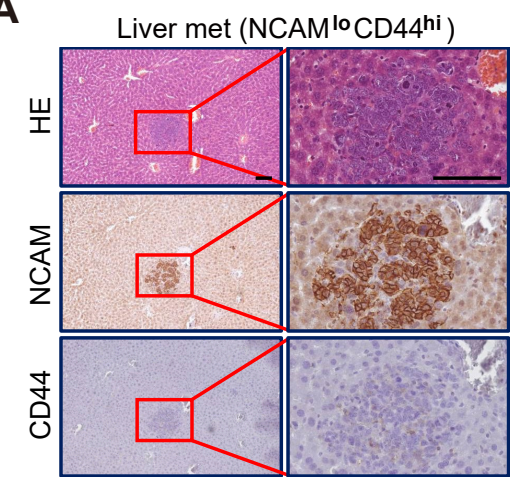

C

GFP

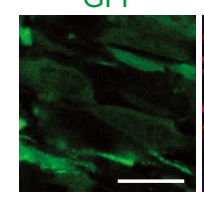

CD44/DAP
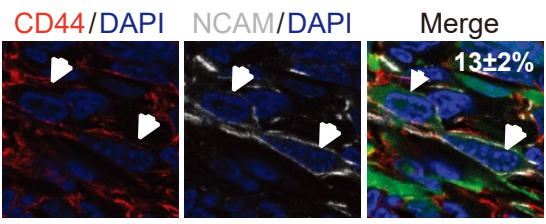

E

GFP

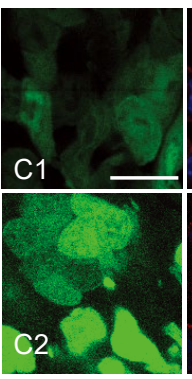

B

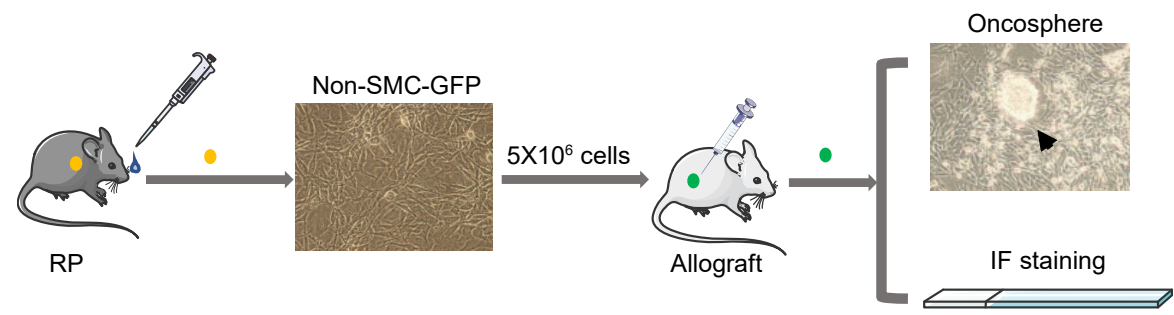

D

Oncosphere
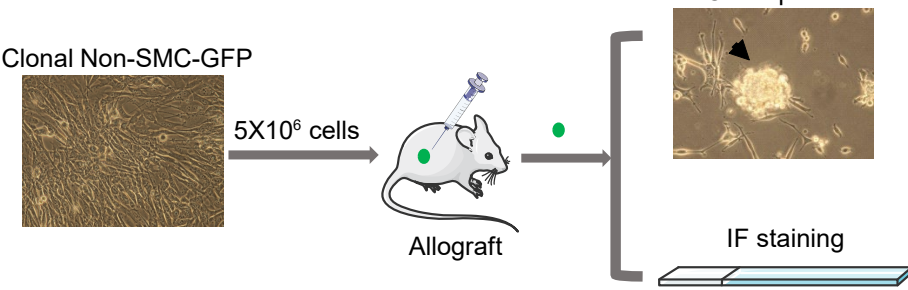

G
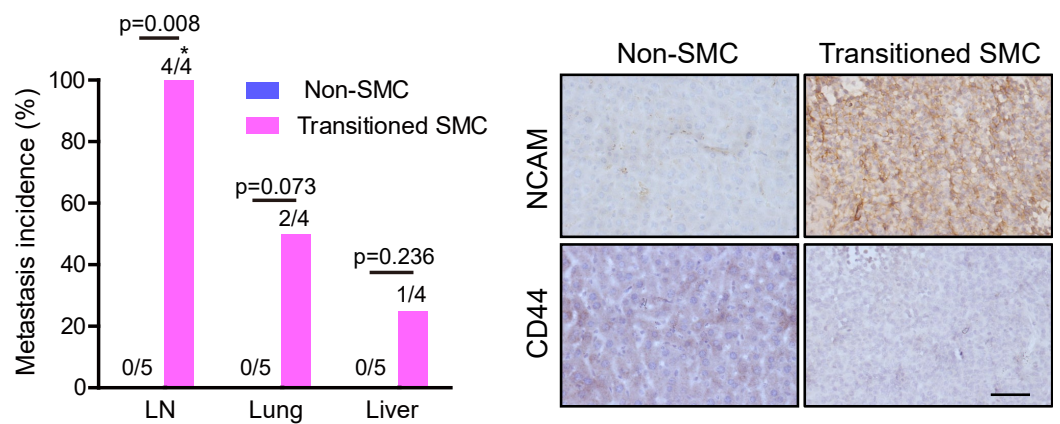
Figure 3

bioRxiv preprint doi: https://doi.org/10.1101/2021.07.28.454244; this version posted July 29, 2021. The copyright holder for this preprint (which was not certified by peer review) is the author/funder. All rights reserved. No reuse allowed without permission.

A

SCN signature-related pathways enriched in SMC

Neurotransmitter secretion Voltage gated potassium channel activity Regulation of neuronal synaptic plasticity Neurotransmitter receptor activity Synaptic signaling Presynapse Synaptic membrane Neurotransmitter transport Neuron fate specification Neuron fate commitment

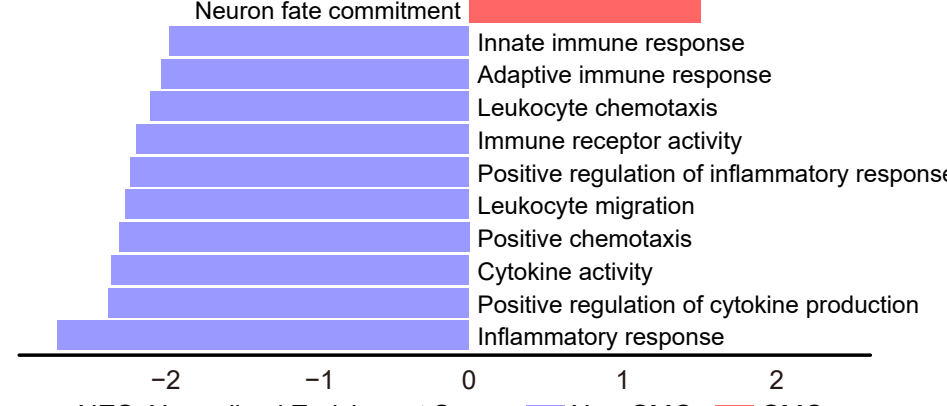

NES: Normalized Enrichment Score. Non-SMC SMC
B
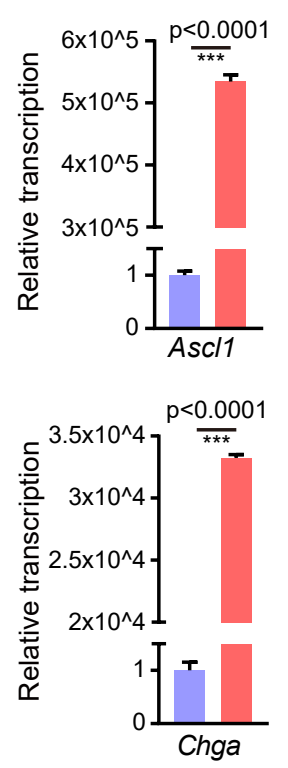

Non-SMC
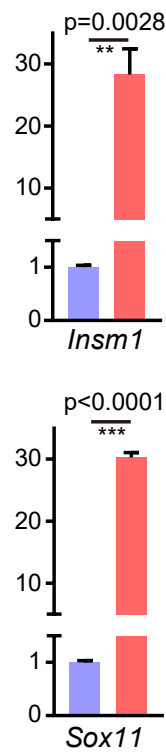

- SMC
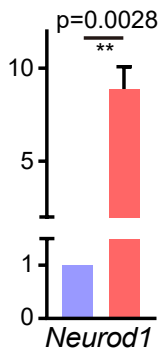

-

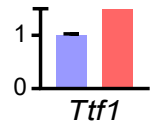

C
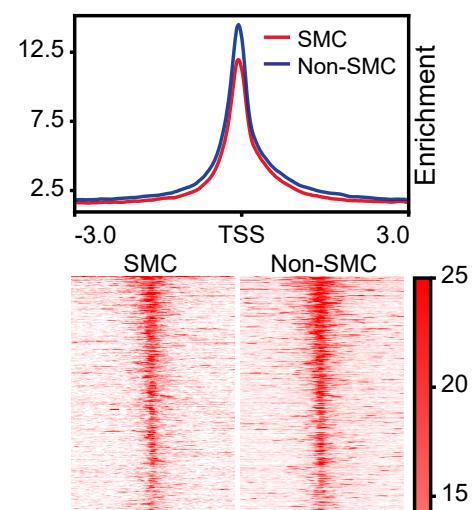

$15 \mathbf{G}$

3ं0 Tśs 30 "3.0 Tśs 3.0 gene distance $(\mathrm{Kb})$
D

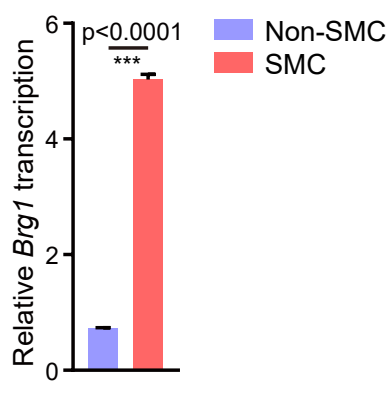

E

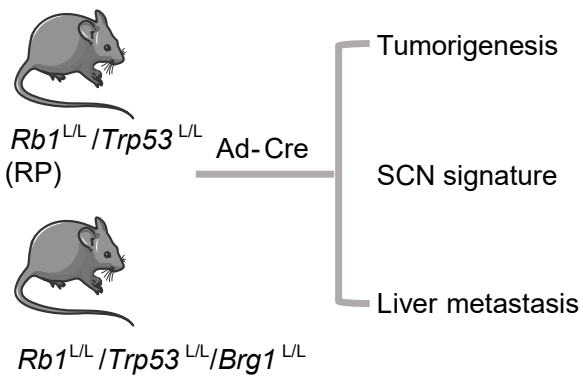

(RPB)
$\mathbf{F}$

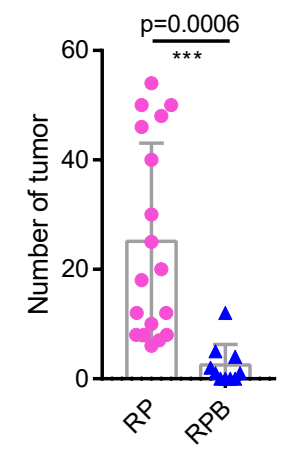

H

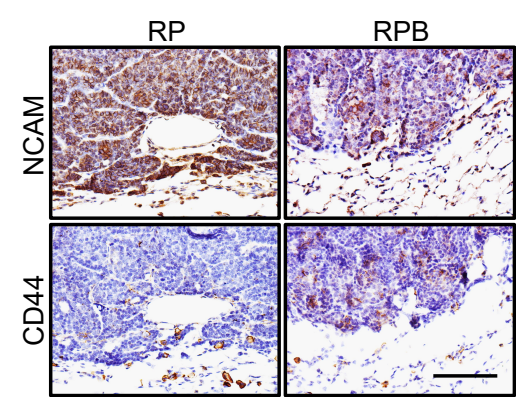

I

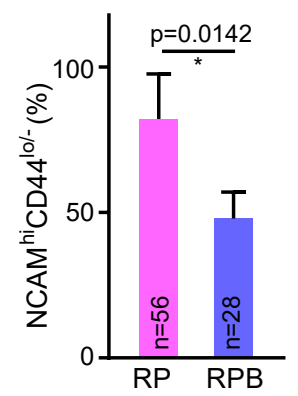

$\mathbf{J}$

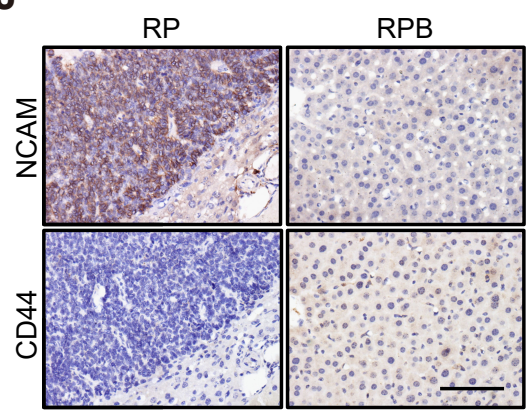

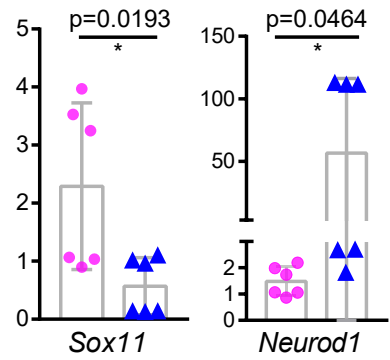
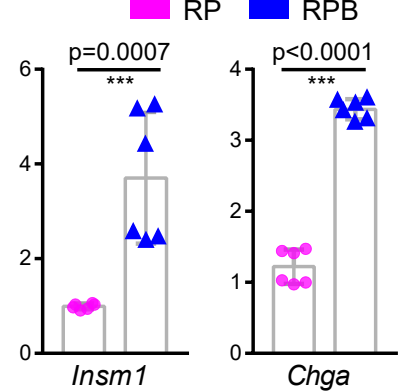

K

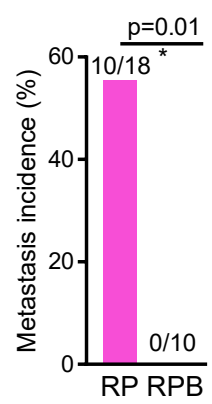


Figure 4

bioRxiv preprint doi: https://doi.org/10.1101/2021.07.28.454244; this version posted July 29, 2021. The copyright holder for this preprint (which

A was not certified by peer review) is the author/funder. All rights reser
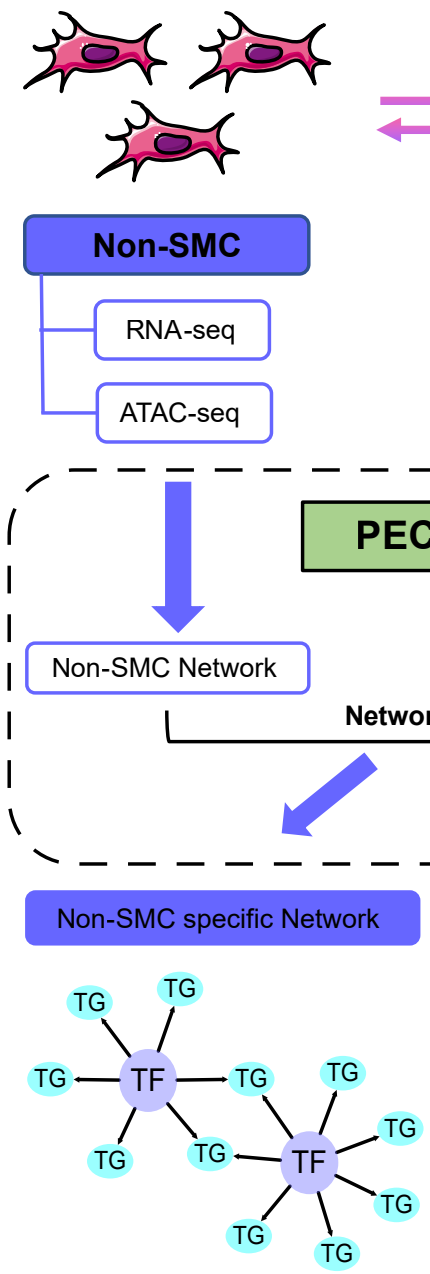

D

TFs and cofactors in Hippo pathway
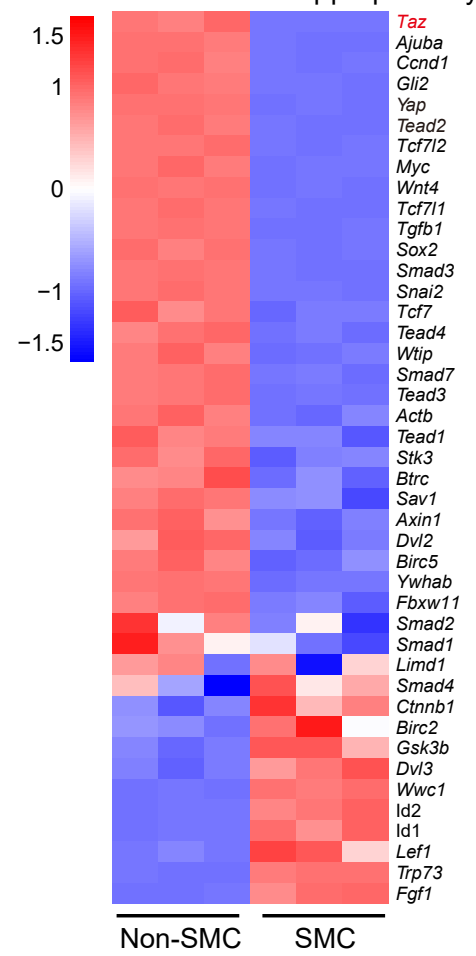
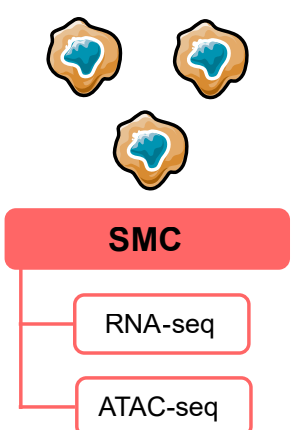

PECA2 Model

etwork Comparison
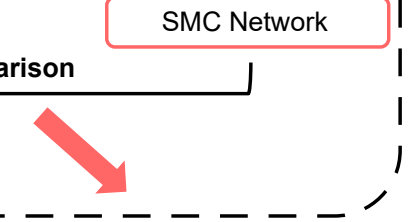

SMC specific Network

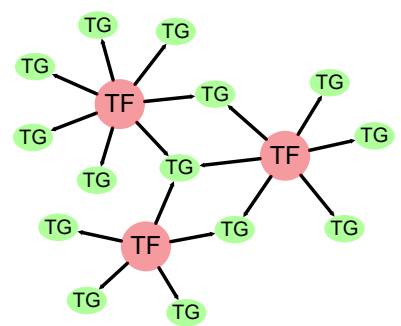

Tead2

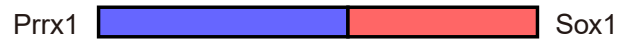

Prrx2 $\square$ Hdac2

Bhlhe40 $\square$ Id1

Nfic $\square$ Tcf4

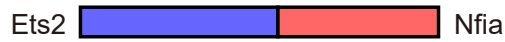

$\mathrm{Nfe} 2 \mathrm{I} 2 \square$ Sox4

Tead1 $\square$ Id 2

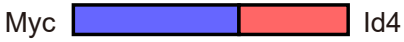

Hmgn3 $\square$ Tcf12

$\begin{array}{lllllll}150 & 100 & 50 & 0 & 200 & 400 & 600\end{array}$

Number of nodes in specific gene regulatory network

$\square$ Enriched in Non-SMC $\square$ Enriched in SMC

C

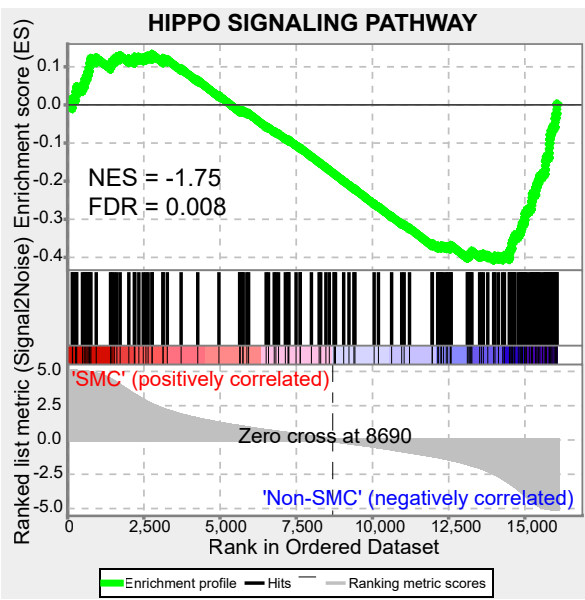

E

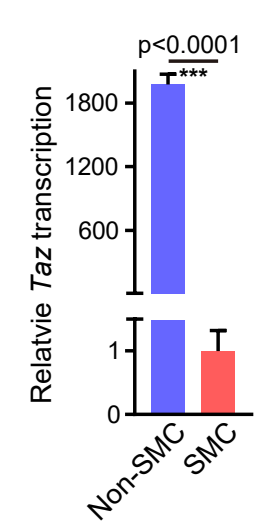

H

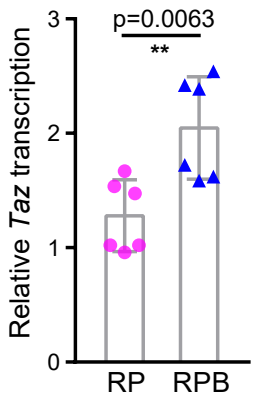

$\mathbf{F}$

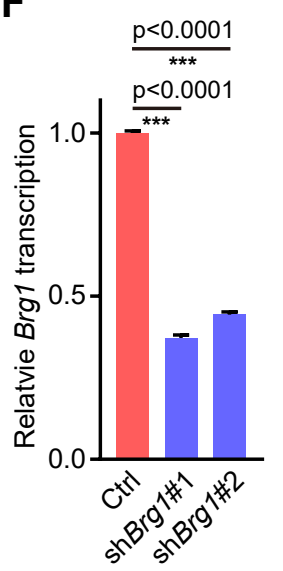

I

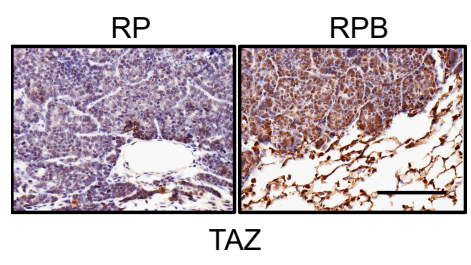

G

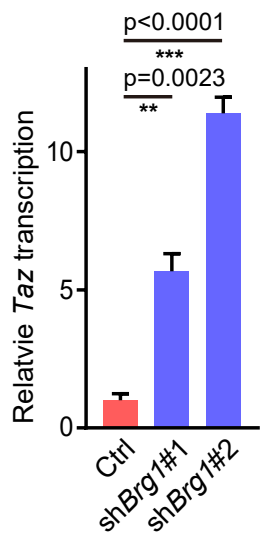

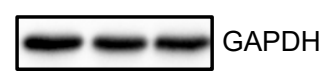

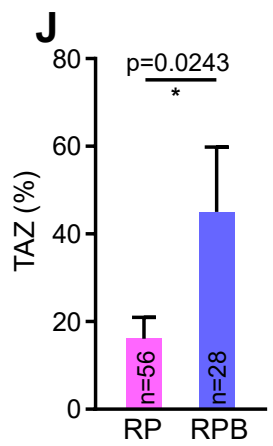


Figure 5 bioRxiv preprint doi: https://doi.org/10.1101/2021.07.28.454244; this version posted July 29, 2021. The copyright holder for this preprint (which A was not certified by peer review) is the author/funder. All rights reserved.
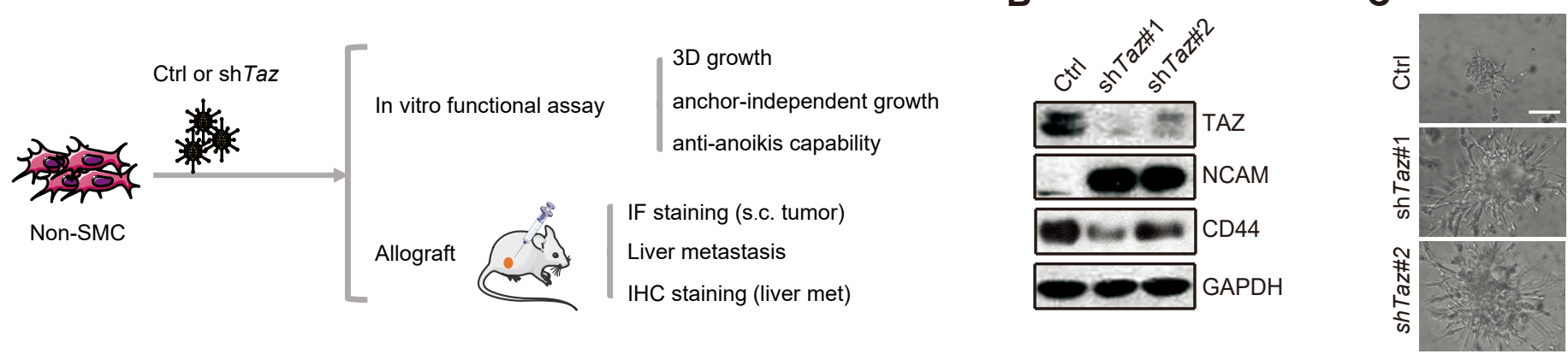

D

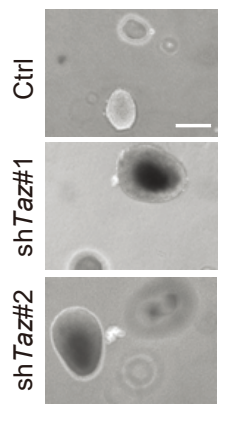

E

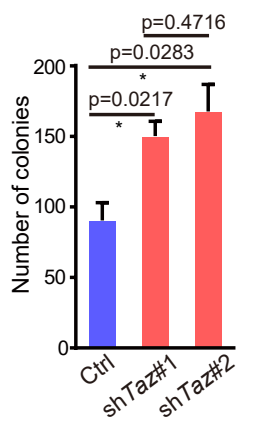

F

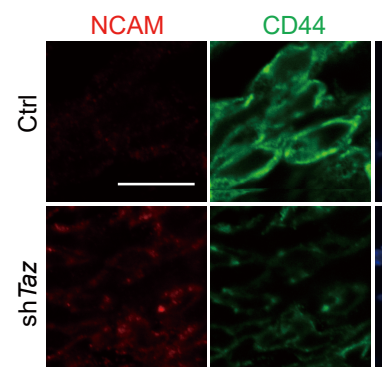

G

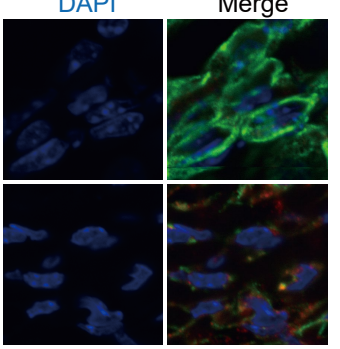

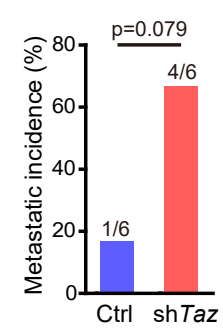

J
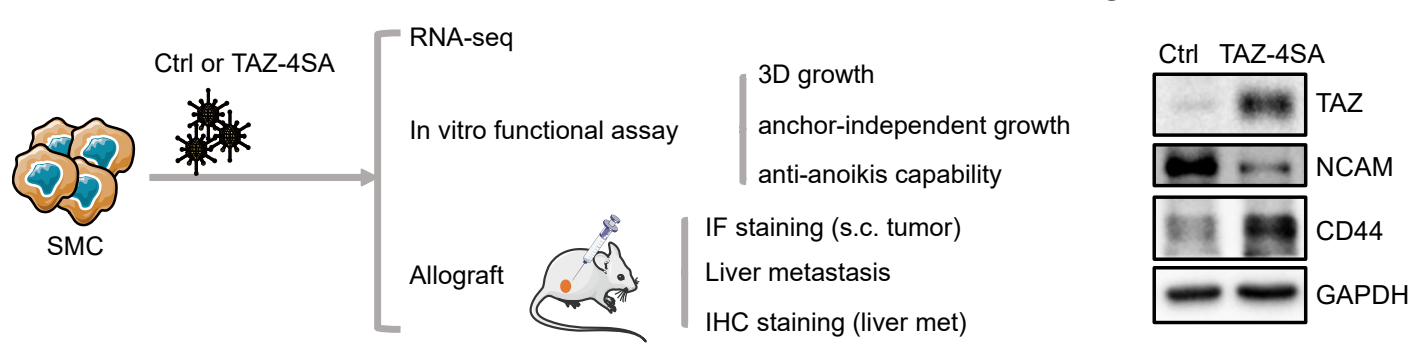

H

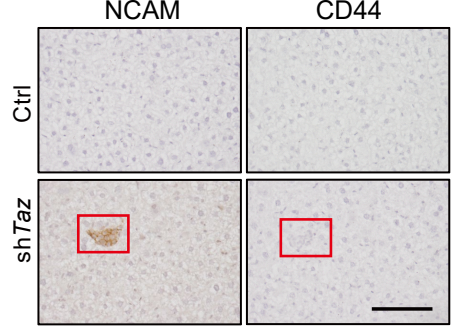

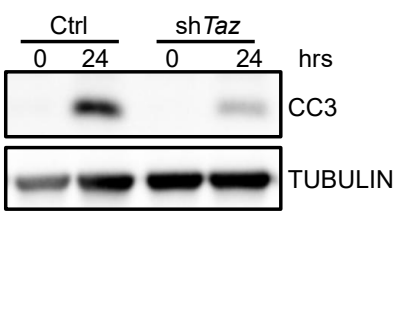

K

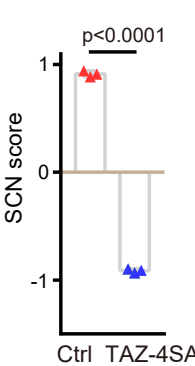

L

0

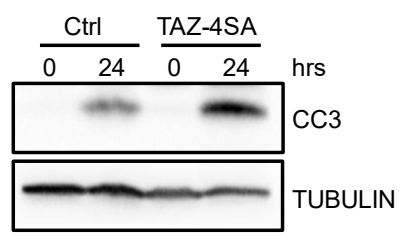

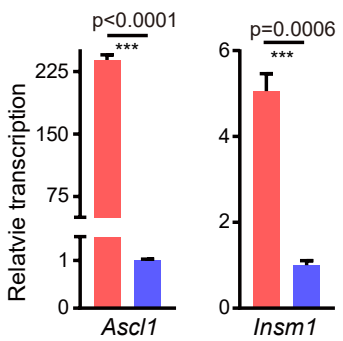
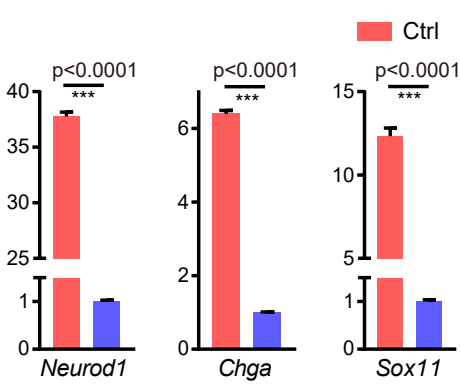

$\mathbf{P}$

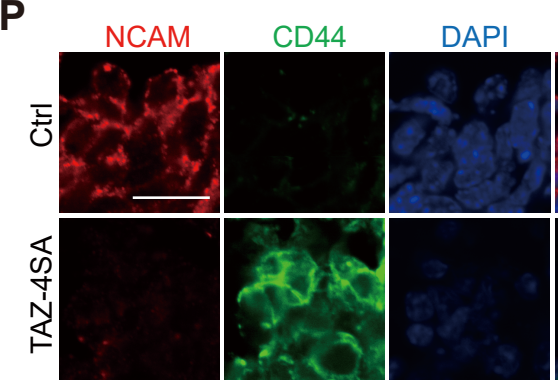

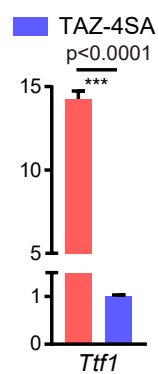

M

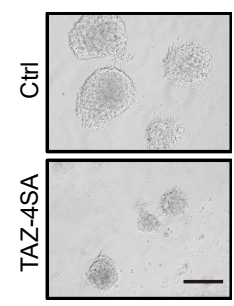

Q

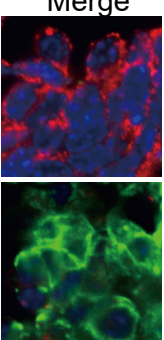

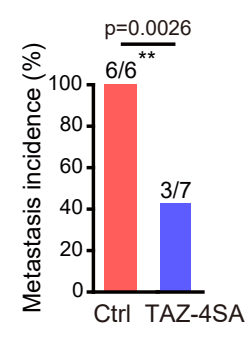

N
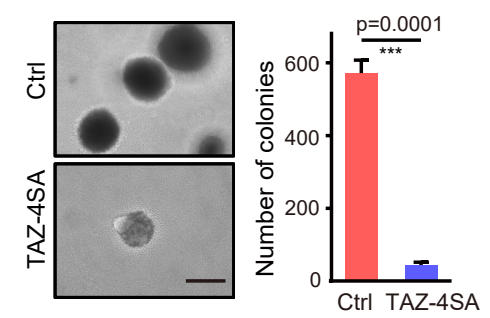

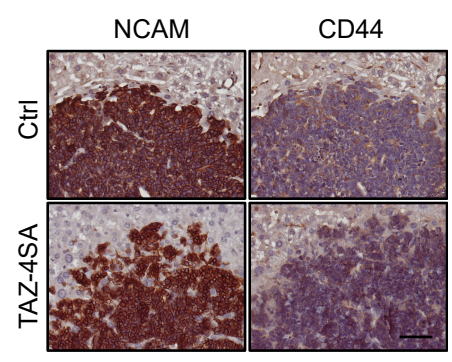


Figure 6

bioRxiv preprint doi: https://doi.org/10.1101/2021.07.28.454244; this version posted July 29, 2021. The copyright holder for this preprint (which was not certified by peer review) is the author/funder. All rights reserved. No reuse allowed without permission.

A

Human SCLC analyses

RNA-seq data (EGAS00001000925 \&

EGAS00001000334) Sc-RNA-seq data of
metastasis

(GSM4558305)

IHC staining in 101 surgical specimens

$\mathrm{SCN}$ signature

SMC existance

Prognosis
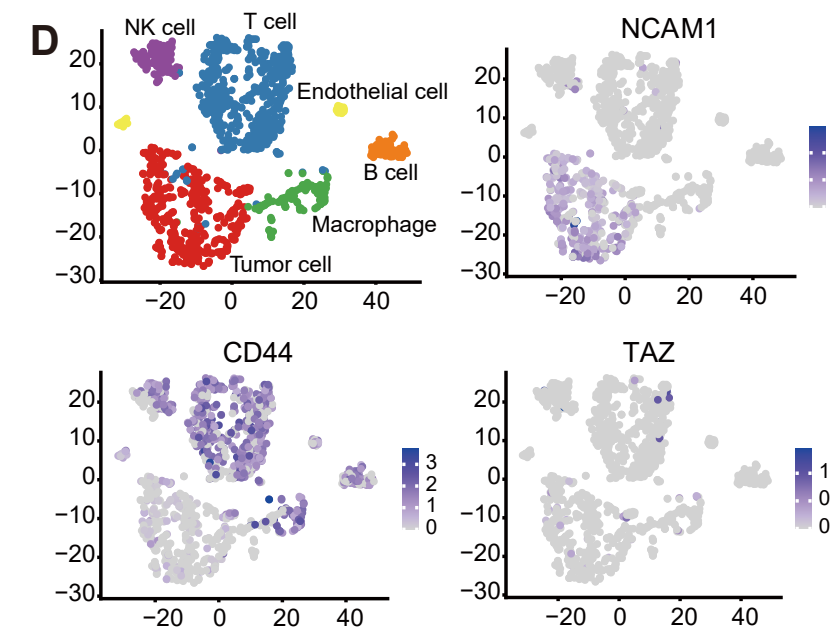

INSM1
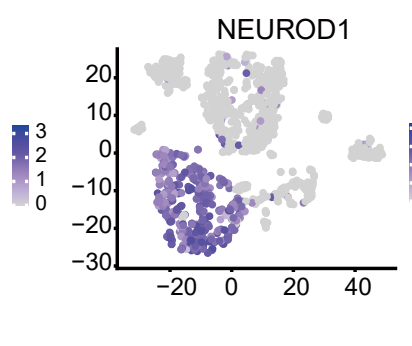

tSNE_1

$F$
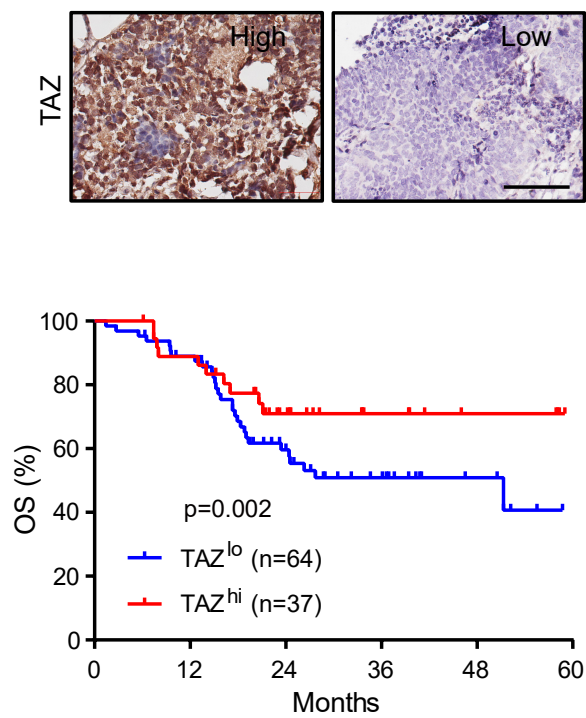

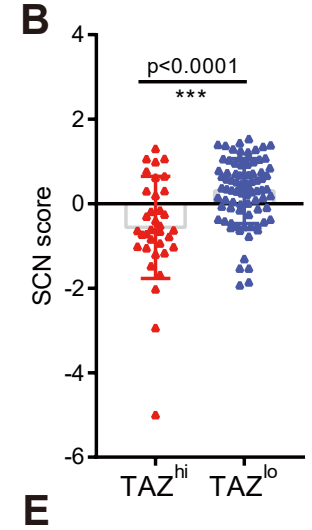

C

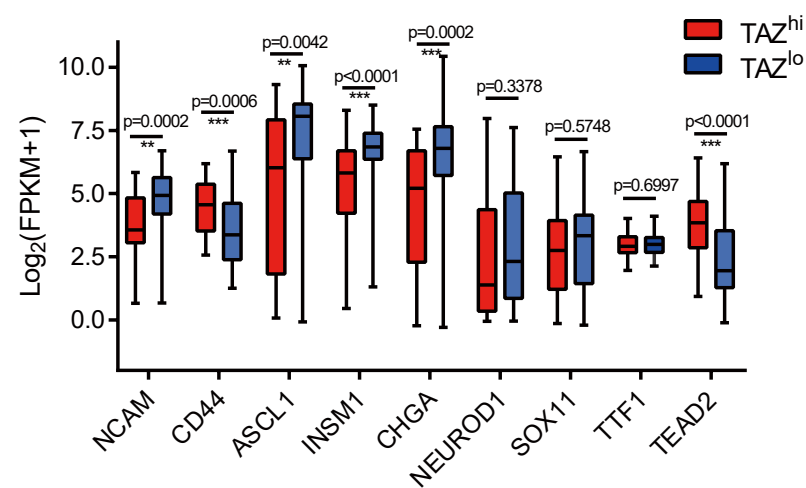

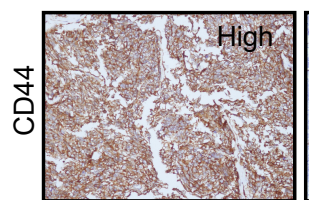
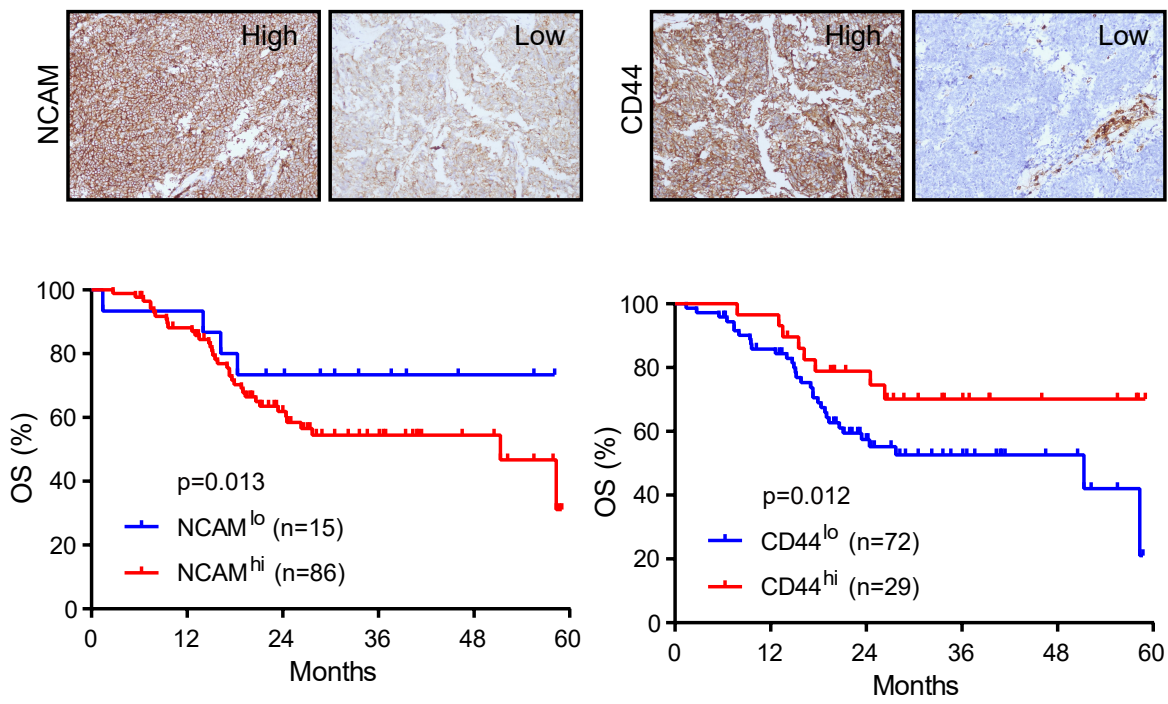

G
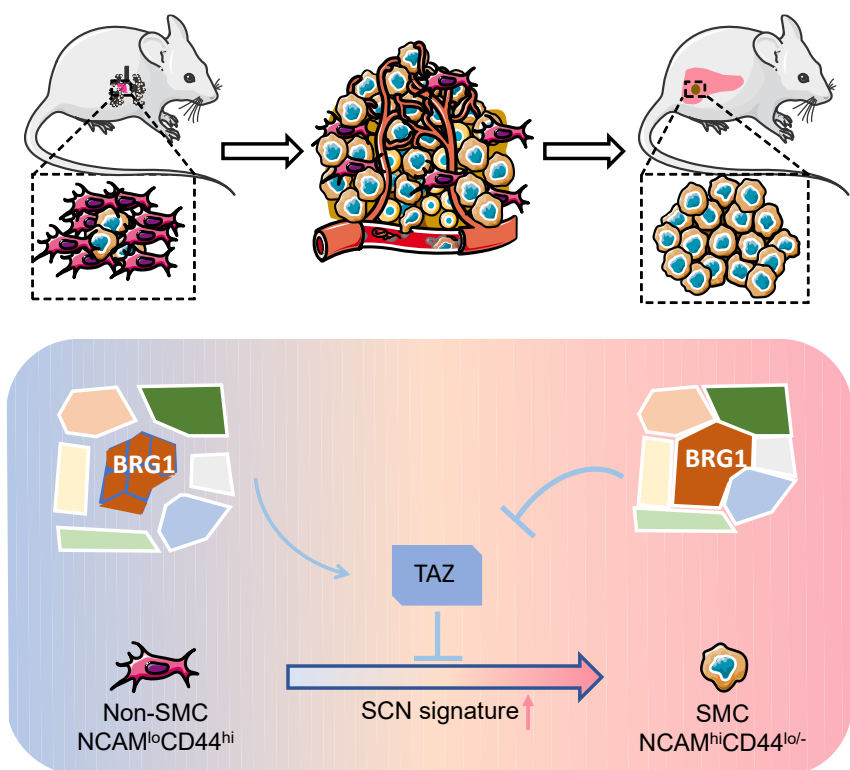